\title{
On the Stanley Ring of a Cubical Complex
}

\author{
G. Hetyei* \\ Department of Mathematics, Massachusetts Institute of Technology, \\ Cambridge, MA 02139, USA
}

\begin{abstract}
We investigate the properties of the Stanley ring of a cubical complex, a cubical analogue of the Stanley-Reisner ring of a simplicial complex. We compute its Hilbert series in terms of the $f$-vector, and prove that by taking the initial ideal of the defining relations, with respect to the reverse lexicographic order, we obtain the defining relations of the Stanley-Reisner ring of the triangulation via "pulling the vertices" of the cubical complex. Applying an old idea of Hochster we see that this ring is Cohen-Macaulay when the complex is shellable, and we show that its defining ideal is generated by quadrics when the complex is also a subcomplex of the boundary complex of a convex cubical polytope. We present a cubical analogue of balanced Cohen-Macaulay simplicial complexes: the class of edge-orientable shellable cubical complexes. Using Stanley's results about balanced Cohen-Macaulay simplicial complexes and the degree two homogeneous generating system of the defining ideal, we obtain an infinite set of examples for a conjecture of Eisenbud, Green, and Harris. This conjecture says that the $h$-vector of a polynomial ring in $n$ variables modulo an ideal which has an $n$-element homogeneous system of parameters of degree two, is the $f$-vector of a simplicial complex.
\end{abstract}

\section{Introduction}

This paper is about some properties of the Stanley ring of a cubical complex. This ring is one of the possible cubical analogues of the Stanley-Reisner ring of a

\footnotetext{
* On leave from the Mathematical Research Institute of the Hungarian Academy of Sciences. Current address: LACIM, Département de mathématiques, Université du Québec à Montréal, Case postale 8888, succursale Centre-Ville, Montréal, Québec, Canada H3C 3P8.
} 
simplicial complex. While in the simplicial case commutative algebra was instrumental in obtaining combinatorial inequalities, this time combinatorics seems to give some commutative algebraic insight.

In Section 1 we introduce the basic motions and define a way of associating simplicial complexes to cubical complexes, such that in the case of convex cubical polytopes our definition coincides with the concept of "triangulation via pulling the vertices." We call our operation in the same way.

In Section 2 we show how to reduce greatly the number of relations defining the Stanley ring $K[\mathscr{C}]$ for all cubical complexes $\mathscr{E}$, and we compute the Hilbert series of $K[\mathscr{C}]$.

In Section 3 we establish a connection between the Stanley ring of a cubical complex and the Stanley-Reisner ring of its triangulations via pulling the vertices: we show that the face ideal of the triangulation via pulling the vertices: we show that the face ideal of the triangulation via pulling the vertices is the initial ideal with respect to the reverse lexicographic order of the face ideal of our cubical complex. This fact is analogous to Sturmfels' result in [22] on initial ideals of toric ideals.

In Section 4 we take a closer look at shellable cubical complexes and their Stanley rings. Using an idea of Hochster we establish the Cohen-Macaulay property of the rings. For later use in Section 5, we prove that the edge-graph of a shellable cubical complex is bipartite.

Section 5 contains the hardest theorem in this paper. We show that in the case of shellable subcomplexes of the boundary complex of a convex cubical polytope, the Stanley ring may be defined by homogeneous relations of degree two. By the theorem of Bruggeser and Mani on the shellability of the boundary complex of convex polytopes [4], our result applies to the entire boundary complex of a convex cubical polytope.

In Section 6 we introduce the notion of edge-orientable cubical complexes, which turns out to be a cubical analogue of completely balanced simplicial complexes. Not only does their Stanley ring contain an explicitly constructible linear system of parameters but they also have a completely balanced triangulation.

Using almost all the previous results of the paper, in Section 7 we construct infinitely many examples verifying a commutative algebraic conjecture of Eisenbud, Green, and Harris. According to this conjecture, the $h$-vector of a polynomial ring in $n$ variables, modulo an ideal which has an $n$-element homogeneous system of parameters of degree two, is the $f$-vector of a simplicial complex. Taking the face ideal of the boundary complex of any edge-orientable convex cubical polytope, and factoring out by a set of linear forms which is a system of parameters modulo the face ideal we obtain an example verifying the conjecture. The proof of the Eisenbud-Green-Harris conjecture in this very special case uses a theorem of Stanley on the $h$-vector of completely balanced simplicial complexes, and it does not work, if we drop the condition of edge-orientability. This makes the question interesting, whether convex cubical polytopes with no edge-orientable boundary exist: if yes (and they probably do) it may be a challenging task to verify the Eisenbud-Green-Harris conjecture already to this class of polytopes. 


\section{Preliminaries}

Definition 1. An (abstract) simplicial complex $\Delta$ is a family of sets (called faces) on a vertex set $V$ such that:

(i) $\{v\} \in \Delta$ holds for every $v \in V$.

(ii) if $\sigma \in \Delta$ and $\tau \subseteq \sigma$, then $\tau \in \Delta$.

For every face $\sigma$ we call $|\sigma|-1$ the dimension of $\sigma$. The maximal faces are called facets, their facets are subfacets.

Definition 2. A cubical complex $\mathscr{C}$ is a family of finite sets (called faces) on a vertex set $V$ with the following properties:

(i) For every $\sigma \in \mathscr{E}$ the elements of $\sigma$ can be represented as the vertices of a finite-dimensional cube, where the faces contained in $\sigma$ are exactly the vertex sets of the faces of the cube. (In particular, we have $\varnothing \in \mathscr{C}$.)

(ii) If $\sigma, \tau \in \mathscr{E}$, then $\sigma \cap \tau \in \mathscr{B}$.

For every face $\sigma$ we call the dimension of the cube associated to $\sigma$ the dimension of $\sigma$. As before, maximal faces are called facets, their facets are subfacets. The one-dimensional faces are also called edges and two vertices $u, v \in V$ are called adjacent if $\{u, v\}$ is an edge. Given a face $\sigma \in \mathscr{C}$ we denote the subcomplex $\{\tau \in \mathscr{C}$ : $\tau \subseteq \sigma\}$ by $\left.\mathscr{E}\right|_{\sigma}$ and call it the restriction of $\mathscr{E}$ to $\sigma$. Moreover, for every nonempty face $\sigma$ we call the complex $\left.\mathscr{C}\right|_{\sigma} \backslash\{\sigma\}$ the boundary of $\sigma$ and we denote it by $\partial(\sigma)$.

It is a well known fact that every simplicial complex has a geometric realization $\phi$ : $V \rightarrow \mathbb{R}^{n}$ in a Euclidean space, such that for every $\sigma \in \Delta \backslash\{\varnothing\}$ the set conv $(\phi(\sigma))$ is a nondegenerate geometric simplex with vertex set $\phi(\sigma)$, and any pair of faces $\sigma, \tau \in \Delta$ we have conv $(\phi(\sigma)) \cap \operatorname{conv}(\phi(\tau))=\operatorname{conv}(\phi(\sigma \cap \tau))$. (See, for example, p. 110 of [17].) It is not necessarily true, however, that cubical complexes have a similar geometric realization $\phi: V \rightarrow \mathbb{R}^{n}$. (Now of course we would require $\operatorname{conv}(\phi(\sigma))$ to be a $\operatorname{dim}(\sigma)$-dimensional polytope with vertex set $\phi(\sigma)$ for every $\sigma \in \mathscr{E} \backslash\{\varnothing\}$. An example of a nonrepresentable cubical complex is the complex $\mathscr{E}$ with three squares $F_{1}, F_{2}, F_{3}$ incident in such a way that they form a Möbius strip. The proof of the fact that this complex has no geometric realization is implicit in the proof of Theorem 7.)

Nevertheless, it is still true even for cubical complexes that their faces may be represented in a standard way.

Definition 3. The geometric standard $n$-cube is the convex polytope

$$
[0,1]^{n}=\left\{\left(x_{1}, x_{2}, \ldots, x_{n}\right) \in \mathbb{R}^{n}: 0 \leq x_{i} \leq 1\right\} \text {. }
$$

We define the (abstract) standard $n$-cube $\mathscr{E}^{n}$ to be the vertex set of $[0,1]^{n}$ together with the inherited face-structure on the vertices. We call any $2^{n}$-element set with an 
isomorphic face-structure an $n$-dimensional cube. We call a geometric realization $\phi: \mathscr{C}^{n} \rightarrow \mathbb{R}^{n}$ standard if we have $\phi\left(V\left(\mathscr{C}^{n}\right)\right)=\{0,1\}^{n}$.

Simplicial complexes occur as triangulations of cubical complexes via pulling the vertices. We define these triangulations in an abstract way, i.e., our operation associates a simplicial complex to even those cubical complexes which have no geometric realizations. For this we use the notion of cubical span.

Definition 4. For a set of vertices $\mathrm{X} \subset \mathrm{V}$ in a cubical complex $\mathscr{E}$ we define the cubical span Cspan $(X)$ of $X$ to be the smallest face containing the set $X$. (If there is no such face, then we leave $\operatorname{Cspan}(X)$ undefined.) For a pair of vertices $\{u, v\}$ and a face $\tau \in \mathscr{C}$ satisfying $\operatorname{Cspan}(\{u, v\})=\tau$ we say that $\{u, v\}$ is a diagonal of $\tau$.

Definition 5. Let $\mathscr{C}$ be a cubical complex on the vertex set $V$ and let $<$ be a linear order on $V$. Let us denote the smallest vertex of a face $\sigma \in \mathscr{C} \backslash\{\varnothing\}$ by $\delta_{<}(\sigma)$. We define the triangulation of $\mathscr{C}$ via pulling the vertices in order $<$ to be the family of all sets $\left\{v_{1}, \ldots, v_{k}\right\}$ such that $k \in \mathbb{N}, v_{1}>\cdots>v_{k}$, and for $i=1, \ldots, k$ we have

$$
v_{i}=\delta<\left(\operatorname{Cspan}\left(\left\{v_{1}, \ldots, v_{i}\right\}\right)\right) \text {. }
$$

Remark. Definition 5 may be naturally extended to the face complex of a convex polytope $\mathscr{P}$ by replacing the term $\operatorname{Cspan}\left(\left\{v_{1}, \ldots, v_{i}\right\}\right)$ with $\operatorname{Pspan}\left(\left\{v_{1}, \ldots, v_{i}\right\}\right)$, where Pspan $(X)$ stands for the smallest face containing a set of vertices $X$. (To be read as the polyhedral span of the set $X$.) In [20] Stanley gives an apparently different definition for triangulations $\Delta_{<}(\mathscr{P})$ of convex polytopes $\mathscr{P}$ via pulling the vertices, but the two definitions may be shown to be equivalent, without much difficulty. It is straightforward from Definition 5 that the restriction of $\Delta_{<}(\mathscr{C})$ to a face $\sigma$ is just the triangulation of $\left.\mathscr{C}\right|_{\sigma}$ via pulling the vertices with respect to the order induced by $<$ on $\sigma$. Hence by Lemma 1.1 of [20], whenever a cubical complex $\mathscr{E}$ is geometrically represented, its (abstract) triangulation via pulling the vertices $\Delta_{<}(\mathscr{C})$ will induce an actual geometric triangulation.

\section{Definition and Elementary Properties of the Stanley Ring}

Stanley suggested investigating the following ring associated to cubical complexes.

Definition 6. Let $\mathscr{C}$ be a cubical complex, and let $K$ be a field. Associate a variable $x_{\nu}$ to each vertex $v \in V$. The Stanley ring $K[\mathscr{E}]$ of the complex $\mathscr{E}$ over the field $K$ is the factor ring $K\left[x_{v}: v \in V\right] / I(\mathscr{C})$, where the ideal $I(\mathscr{E})$ is generated by the following elements:

(i) $x_{v_{1}} \cdot x_{v_{2}} \cdots x_{v_{k}}$ for all $v_{1}, \ldots, v_{k} \in V$ such that $\left\{v_{1}, \ldots, v_{k}\right\}$ is not contained in any face of $\mathscr{E}$.

(ii) $x_{u} \cdot x_{\nu}-x_{u^{\prime}} \cdot x_{v^{\prime}}$ for all $u, u^{\prime}, v, v^{\prime} \in V$ such that $\{u, v\}$ and $\left\{u^{\prime}, v^{\prime}\right\}$ are diagonals of the same face $\operatorname{Cspan}(\{u, v\})=\operatorname{Cspan}\left(\left\{u^{\prime}, v^{\prime}\right\}\right) \in \mathscr{C}$. 
We denote the ideal generated by the elements of types (i) and (ii) by $I_{1}(\mathscr{E})$ and $I_{2}(C)$, respectively. We call $I(\mathscr{E})$ the face ideal of the cubical complex $\mathscr{C}$.

In this section we show that condition (i) can be weakened to requiring the product of at most three variables to be in $I(\mathscr{C})$, whenever the set of their indices is not contained in any face. In Section 5 we prove that for an important class of cubical complexes (shellable subcomplexes of boundary complexes of convex cubical polytopes), it is even sufficient to set the product of pairs to be zero in $K(\mathscr{C})$ when they are not diagonals of a face. In doing so, the following equivalence relation defined on multisets of vertices is instrumental. (Entries between brackets "l" and "J" are to be read as a list of elements of a multiset.)

Definition 7. We call the multisets of vertices $\left\lfloor u_{1}, u_{2}, \ldots, u_{k}\right\rfloor$ and $\left\lfloor v_{1}, v_{2}, \ldots, v_{l}\right\rfloor$ equivalent, if $k=l$ and $\left\lfloor v_{1}, v_{2}, \ldots, v_{k}\right\rfloor$ can be obtained from $\left\lfloor u_{1}, u_{2}, \ldots, u_{k}\right\rfloor$ by repeated application of the following operation. If Cspan $\left(\left\{u_{1}, u_{2}\right\}\right)$ exists, replace $\left\lfloor u_{1}, u_{2}, u_{3}, \ldots, u_{k}\right\rfloor$ with $\left\lfloor u_{1}^{\prime}, u_{2}^{\prime}, u_{3}, \ldots, u_{k}\right\rfloor$, where $\left\lfloor u_{1}^{\prime}, u_{2}^{\prime}\right\rfloor$ is any diagonal of $\operatorname{Cspan}\left(\left\{u_{1}, u_{2}\right\}\right)$.

The operation of replacing a diagonal with another one is reversible, and so we defined in deed an equivalence relation. Clearly, if a face $\tau \in \mathscr{C}$ contains $\left\{u_{1}, \ldots, u_{k}\right\}$, then the same holds for all equivalent multisets $\left\lfloor v_{1}, \ldots, v_{k}\right\rfloor$. Hence we can say that a face $\tau$ contains or does not contain a given equivalence class of multisets. In particular, Cspan $\left(\left\lfloor u_{1}, u_{2}, \ldots, u_{k}\right\rfloor\right)$ is simultaneously defined or not defined for all multisets of an equivalence class, and its value is constant on an equivalence class, on which it is defined. The definitions yield immediately the following connections between the equivalence classes of multisets and monomials.

Lemma 1. The monomials of $K\left[x_{v}: v \in V\right]$ have the following properties:

1. We have $x_{u_{1}} \cdots x_{u_{k}} \in I_{1}(\mathscr{E})$ if and only if $C \operatorname{span}\left(\left\lfloor u_{1}, \ldots, u_{k}\right\rfloor\right)$ does not exist.

2. The differences $x_{\underline{u}}-x_{\underline{\underline{v}}}$, where $\underline{u}$ and $\underline{v}$ are equivalent multisets of vertices, form a generating system of the $K$-vector space $I_{2}(\mathscr{C})$. Consequently, monomials of degree $k$ indexed by equivalent multisets of vertices represent the same element modulo $I_{2}(\mathscr{C})$.

The following theorem is the key to understanding the role of the equivalence of multisets of vertices.

Theorem 1. Monomials not belonging to $I_{1}(\mathscr{C})$ and associated to multisets from different equivalence classes are linearly independent modulo $I(\mathscr{C})$.

Proof. Assume that we have a linear combination $\Sigma_{v} \lambda_{v} \cdot x_{\underline{v}} \in I(\mathscr{E})$ of monomials $x_{\underline{v}} \notin I_{1}(\mathscr{E})$, with coefficients $\lambda_{\underline{v}} \in K$, such that all the multisets $\underline{v}=\left\lfloor v_{1}, \ldots, v_{l}\right\rfloor$ occurring in this sum belong to different equivalence classes. Let us fix one $x_{\underline{u}}=x_{u_{1}} \cdots x_{u_{k}}$, and show that we must have $\lambda_{\underline{u}}=0$. By $x_{\underline{u}} \notin I_{1}(\mathscr{C})$ the face 
$\operatorname{Cspan}\left(\left\lfloor u_{1}, \ldots, u_{k}\right\rfloor\right)$ must exist. The factor of $K[\mathscr{E}]$ by the ideal $\left(x_{v}: v \notin\right.$ Cspan $\left.\left(\left\lfloor u_{1}, \ldots, u_{k}\right\rfloor\right)\right)$ is the Stanley ring of $\left.\mathscr{C}\right|_{\operatorname{Cspan}\left(\left\lfloor u_{1}, \ldots, u_{k}\right\rfloor\right)}$, and we have $x_{\underline{u}} \notin$ $I_{1}\left(\left.\mathscr{E}\right|_{\text {Cspan }\left(\left\lfloor u_{1}, \ldots, u_{k}\right\rfloor\right)}\right)$. Moreover, if two multisubsets of $V\left(\left.\mathscr{E}\right|_{\left.C \operatorname{span}\left(\mid u_{1}, \ldots, u_{k}\right\rfloor\right)}\right)$ are not equivalent in $\mathscr{E}$, then they are not equivalent in $\left.\mathscr{C}\right|_{\operatorname{Cspan}\left(\left[u_{1}, \ldots, u_{k}\right\rfloor\right)}$ either. Thus without loss of generality we may assume $\mathscr{C}=\left.\mathscr{C}\right|_{C \operatorname{cspan}\left(\left|u_{1}, \ldots, u_{k}\right|\right)}$, i.e., that $\mathscr{C}$ is a standard $n$-cube $\mathscr{C}^{n}$ for some $n \in \mathbb{N}$.

$I_{1}\left(\mathscr{C}^{n}\right)=0$ implies $I\left(\mathscr{C}^{n}\right)=I_{2}\left(\mathscr{C}^{n}\right)$. Let us fix a standard geometric representation $\phi$ of $\mathscr{C}^{n}$, and consider the $K$-algebra homomorphism $\alpha: K\left[x_{v}: v \in V\left(\mathscr{C}^{n}\right)\right] \rightarrow$ $K\left[y_{0}, y_{1}, \ldots, y_{n}\right]$ defined by $\underline{\alpha}\left(x_{v}\right)=y_{0} \cdot y_{1}^{\phi_{1}(v)} \ldots y_{n}^{\phi_{n}(v)}$. The kernel of $\underline{\alpha}$ obviously contains all binomials of the form $x_{u} \cdot x_{v}-x_{u^{\prime}} \cdot x_{v^{\prime}}$, where Cspan $(\{u, v\})=$ Cspan $\left(\left\{u^{\prime}, v^{\prime}\right\}\right)$. Hence Ker $\underline{\alpha}$ contains $I\left(\mathscr{C}^{n}\right)=I_{2}\left(\mathscr{C}^{n}\right)$, and monomials associated to equivalent multisets are mapped into the same monomial under $\underline{\alpha}$. Therefore in order to prove $\lambda_{\underline{u}}=0$, we only need to show that for a multiset $\underline{v}=\left\lfloor v_{1}, \ldots, v_{l}\right\rfloor$ not equivalent to $\underline{u}$ we have $\underline{\alpha}\left(x_{v}\right) \neq \underline{\alpha}\left(x_{\underline{u}}\right)$, since then the coefficient of the monomial $\underline{\alpha}\left(x_{\underline{u}}\right)$ in $0=\Sigma_{\underline{\underline{v}}} \lambda_{\underline{\underline{v}}} \cdot \underline{\alpha}\left(x_{\underline{v}}\right)$ will be $\lambda_{\underline{\underline{u}}}$.

Let us denote by $\operatorname{Set}(v)$ the set $\left\{i: \phi_{i}(v)=1\right\}$. The operation Set is a bijection between $V\left(\mathscr{C}^{n}\right)$ and the subsets of $\{1,2, \ldots, n\}$. A subset $X$ of $V\left(\mathscr{C}^{n}\right)$ is a face iff $\{\operatorname{Set}(v): v \in X\}$ is an interval of the boolean algebra $P(\{1,2, \ldots, n\})$. Hence we have $\operatorname{Cspan}(\{u, v\})=\operatorname{Cspan}\left(\left\{u^{\prime}, v^{\prime}\right\}\right)$ iff for the corresponding subsets $\operatorname{Set}(u) \cap \operatorname{Set}(v)=$ $\operatorname{Set}\left(u^{\prime}\right) \cap \operatorname{Set}\left(v^{\prime}\right)$ and $\operatorname{Set}(u) \cup \operatorname{Set}(v)=\operatorname{Set}\left(u^{\prime}\right) \cup \operatorname{Set}\left(v^{\prime}\right)$ hold. For a monomial $x_{\underline{v}}=x_{v_{1}} \cdots x_{v_{l}}$ we have $\underline{\alpha}\left(x_{\underline{v}}\right)=y_{0}^{\alpha_{0}} \cdot y_{1}^{\alpha_{1}} \cdots y_{n}^{\alpha_{n}}$, where $\alpha_{0}=l$, and for $i \geq 1, \alpha_{i}$ is the number of $j$ 's such that $i \in \operatorname{Set}\left(v_{j}\right)$. (We count repeated vertices with their multiplicity.)

Let $\underline{v}=\left\lfloor v_{1}, \ldots, v_{l}\right\rfloor$ be an arbitrary multiset of vertices. Replacing any pair $\left(v_{i}, v_{j}\right)$ with the pair $\left(\operatorname{Set}^{-1}\left(\operatorname{Set}\left(v_{i}\right) \cap \operatorname{Set}\left(v_{j}\right)\right)\right.$, $\left.\operatorname{Set}^{-1}\left(\operatorname{Set}\left(v_{i}\right) \cup \operatorname{Set}\left(v_{j}\right)\right)\right)$, we obtain an equivalent multiset of vertices. Using this operation repeatedly, we can reach an equivalent multiset $\underline{v}^{\prime}=\left\lfloor v_{1}^{\prime}, \ldots, v_{l}^{\prime}\right\rfloor$ such that $\operatorname{Set}\left(v_{1}^{\prime}\right) \subseteq \cdots \subseteq \operatorname{Set}\left(v_{l}^{\prime}\right)$ holds. (We can prove this by induction on $l$.) Now the statement follows from the obvious fact that for this multiset $\left\lfloor v_{1}^{\prime}, \ldots, v_{l}^{\prime}\right\rfloor$ we must have $\operatorname{Set}\left(v_{j}^{\prime}\right)=\left\{i \in\{1,2, \ldots, n\}: \alpha_{i} \geq\right.$ $l+1-j\}$. Therefore $\underline{\alpha}$ assigns different monomials to different equivalence classes of multisets of vertices.

Corollary 1. We have $x_{u_{1}} \cdots x_{u_{k}} \in I(\mathscr{C})$ if and only if $\operatorname{Cspan}\left(\left\lfloor u_{1}, \ldots, u_{k}\right\rfloor\right)$ does not exist.

Corollary 2. Two monomials $x_{u_{1}} \cdots x_{u_{k}} \notin I(\mathscr{C})$ and $x_{v_{1}} \cdots x_{v_{l}} \notin I(\mathscr{C})$ represent the same class modulo $I(\mathscr{C})$ if and only if $k=l$ and the multisets $\left\lfloor u_{1}, \ldots, u_{k}\right\rfloor$ and $\left\lfloor v_{1}, \ldots, v_{k}\right\rfloor$ are equivalent.

Remark. Theorem 1 is also a straight consequence of the proof of Theorem 4 . We included an elementary proof, such that we may avoid the use of Gröbner basis theory in this section. Part of the argument presented may also be applied to show the following lemma. 
Lemma 2. Let $\mathscr{C}$ be an arbitrary cubical complex and $k \geq 2$. Then any monomial $x_{u_{1}} \cdot x_{u_{2}} \cdots x_{u_{k}}$ such that $\operatorname{Cspan}\left(\left\{u_{1}, \ldots, u_{k}\right\}\right)$ exists, is equivalent modulo $I_{2}(\mathscr{C})$ to a monomial $x_{v_{1}} \cdot x_{v_{2}} \cdots x_{v_{k}}$ such that

$$
\operatorname{Cspan}\left(\left\{v_{1}, v_{2}\right\}\right)=\operatorname{Cspan}\left(\left\{u_{1}, \ldots, u_{k}\right\}\right)=\operatorname{Cspan}\left(\left\{v_{1}, \ldots, v_{k}\right\}\right)
$$

holds.

Proof. Without loss of generality we may assume $\mathscr{C}=\operatorname{Cspan}\left(\left\{u_{1}, \ldots, u_{k}\right\}\right)$, i.e., that $\mathscr{C}$ is a standard $n$-cube $\mathscr{C}^{n}$. Let us fix again a geometric realization $\phi$ and denote by $\operatorname{Set}(v)$ the subset of $\{1,2, \ldots, n\}$ with characteristic vector $\phi(v)$. We have shown in the proof of Theorem 1 that $\left\lfloor u_{1}, \ldots, u_{k}\right\rfloor$ is equivalent to a multiset $\left\lfloor v_{1}, \ldots, v_{k}\right\rfloor$ such that $\operatorname{Set}\left(v_{1}\right) \subseteq \cdots \subseteq \operatorname{Set}\left(v_{k}\right)$ holds. This $\left\lfloor v_{1}, \ldots, v_{k}\right\rfloor$ will have the required properties. (Observe that in the notation of the proof of Theorem $1,\left\{v_{1}, v_{k}\right\}$ will be a diagonal of $\operatorname{Cspan}\left(\left\{u_{1}, \ldots, u_{k}\right\}\right)$, whereas in the notation of the statement of this lemma $\left\{v_{1}, v_{2}\right\}$ is a diagonal. However, the difference is only in the numbering of the vertices, which is irrelevant when we investigate multisets of vertices.)

Using Lemma 2 we can show the following theorem.

Theorem 2. Let $\mathscr{C}$ be an arbitrary cubical complex. Let $I_{1}^{\prime}(\mathscr{E})$ be the ideal of $K\left[x_{v}: v \in V\right]$ generated by all monomials $x_{v_{1}} \cdots x_{v_{k}}$ such that $k \leq 3$, and $\left\{v_{1}, \ldots, v_{k}\right\}$ is not contained in any face of $\mathscr{B}$. Then we have

$$
I(\mathscr{C})=I_{1}^{\prime}(\mathscr{E})+I_{2}(\mathscr{C}) .
$$

Proof. By definition, $I_{1}^{\prime}(\mathscr{C})$ is contained in $I_{1}(\mathscr{C})$. Hence it is sufficient to show that if $\left\{v_{1}, \ldots, v_{k}\right\}$ is not contained in any face of $\mathscr{C}$, then $x_{v_{1}} \cdots x_{v_{k}}$ is congruent modulo $I_{2}(\mathscr{E})$ to a monomial from $I_{1}^{\prime}(\mathscr{E})$. We prove this statement by induction on $k$. For $k=2,3$ we have $x_{v_{1}} \cdots x_{v_{k}} \in I_{1}^{\prime}(\mathscr{E})$. Assume we know the statement for $k$, and that we are given $v_{1}, v_{2}, \ldots, v_{k+1}$ such that $\left\{v_{1}, \ldots, v_{k+1}\right\}$ is not contained in any face of $\mathscr{C}$. If $\left\{v_{1}, \ldots, v_{k}\right\}$ is not contained in any face, then we have $x_{v_{1}} \cdots x_{v_{k}} \in I_{1}(\mathscr{E})$, by the induction hypothesis we get $x_{v_{1}} \cdots x_{v_{k}} \in I_{1}^{\prime}(\mathscr{E})$, and so $x_{v_{1}} \cdots x_{v_{k}} \cdot x_{v_{k+1}} \in I_{1}^{\prime}(\mathscr{E})$. Hence we may assume that $\operatorname{Cspan}\left(\left\{v_{1}, \ldots, v_{k}\right\}\right)$ exists. By Lemma 2 , the monomial $x_{v_{1}} \cdots x_{v_{k}}$ is congruent modulo $I_{2}(\mathscr{C})$ to a monomial $x_{v_{1}^{\prime}} \cdots x_{v_{k}^{\prime}}$ such that we have

$$
\operatorname{Cspan}\left(\left\{v_{1}^{\prime}, v_{2}^{\prime}\right\}\right)=\operatorname{Cspan}\left(\left\{v_{1}, \ldots, v_{k}\right\}\right) \text {. }
$$

However, then $\operatorname{Cspan}\left(\left\{v_{1}^{\prime}, v_{2}^{\prime}, v_{k+1}\right\}\right)$ does not exist and we get

$$
x_{v_{1}^{\prime}} \cdot x_{v_{2}^{\prime}} \cdot x_{v_{k+1}} \in I_{1}^{\prime}(\mathscr{E}) .
$$

This implies

$$
x_{v_{1}^{\prime}} \cdots x_{v_{k}^{\prime}} \cdot x_{v_{k+1}} \in I_{1}^{\prime}(\mathscr{E}) \text {, }
$$

and so $x_{v_{1}} \cdots x_{v_{k}} \cdot x_{v_{k+1}}$ is congruent modulo $I_{2}(\mathscr{C})$ to an element of $I_{1}^{\prime}(\mathscr{E})$. 
Theorem 1 and its corollaries also allow us to compute the Hilbert series of the Stanley ring of a cubical complex. Recall that the Hilbert series of a finitely generated $\mathbb{N}$-graded $K$-algebra $A$ is usually defined as

$$
\mathscr{H}(A, t)=\sum_{n=0}^{\infty} \operatorname{dim}_{K}\left(A_{n}\right) \cdot t^{n}
$$

where $A_{n}$ is the vector space generated by the homogeneous elements of degree $n$, and the operator $\operatorname{dim}_{K}$ stands for taking the vector space dimension. (For details, see, e.g., p. 33 of [21].)

Theorem 3. Let $\mathscr{C}$ be a d-dimensional cubical complex and let $f_{i}$ be the number of $i$-dimensional faces of $\mathscr{E}$. Then the Hilbert series $\mathscr{H}(K[\mathscr{C}], t)$ of the graded algebra $K[\mathscr{E}]$ is given by

$$
\mathscr{X}(K[\mathscr{C}], t)=1+\sum_{i=0}^{d} f_{i} \cdot \sum_{k=1}^{\infty}(k-1)^{i} \cdot t^{k}
$$

Proof. $K[\mathscr{E}]$ may be written as a direct sum of $K$-vector spaces as follows:

$$
K[\mathscr{E}]=\underset{\sigma \in \mathscr{C}}{\oplus} \underset{k=0}{\oplus}\left\langle x_{u_{1}} \cdots x_{u_{k}}: \operatorname{Cspan}\left(\left\lfloor u_{1}, \ldots, u_{k}\right\rfloor\right)=\sigma\right\rangle
$$

(Note that this sum includes the vector space generated by the empty product 1 for $\sigma=\varnothing$ and $k=0$.) It is a consequence of Theorem 1 and its corollaries that for an $i$-dimensional face $\sigma \in \mathscr{C}$ and a positive integer $k$, the dimension of $\left\langle x_{u_{1}} \cdots x_{u_{k}}\right.$ : $\left.\operatorname{Cspan}\left(\left\lfloor u_{1}, \ldots, u_{k}\right\rfloor\right)=\sigma\right\rangle$ is equal to the number of multisets $\left\lfloor X_{1}, \ldots, X_{k}\right\rfloor$ of subsets of $\{1,2, \ldots, i\}$ such that we have

$$
\varnothing=X_{1} \subseteq X_{2} \subseteq \cdots \subseteq X_{k}=\{1,2, \ldots, i\} .
$$

(For $i=0$ we write $\varnothing$ instead of $\{1,2, \ldots, i\}$.) The number of such multisets is 1 for $i=0$, and 0 for $i>0, k=1$. When $i>0$ and $k \geq 2$ then for every $j \in\{1,2, \ldots, i\}$ there is a unique $\beta(j) \in\{1, \ldots, k-1\}$ such that $j \notin X_{1} \cup X_{2} \cup \cdots \cup X_{\beta(j)}$ and $j \in X_{\beta(j)+1} \cap X_{\beta(j)=2} \cap \cdots \cap X_{k}$. The values $\beta(j)$ may be chosen independently, in $(k-1)^{i}$ ways. Thus we have

$$
\operatorname{dim}\left(\left\langle x_{u_{1}} \cdots x_{u_{k}}: \operatorname{Cspan}\left(\left\lfloor u_{1}, \ldots, u_{k}\right\rfloor\right)=\sigma\right\rangle\right)=(k-1)^{i},
$$

and the theorem follows.

Introducing

$$
\Phi_{0}(t) \stackrel{\text { def }}{=} \sum_{k \geq 0} t^{k}=\frac{1}{1-t} \quad \text { and } \quad \Phi_{r}(t) \stackrel{\text { def }}{=} \sum_{k \geq 0} k^{r} \cdot t^{k} \quad \text { for } \quad r \geq 1,
$$

we may rewrite (1) as

$$
\mathscr{X}(K[\mathscr{C}], t)=1+\sum_{i=0}^{d} f_{i} \cdot t \cdot \Phi_{i}(t)
$$


Let $D$ denote the derivation operator of the polynomial ring $\mathbb{Z}[t]$ defined by $D: t \mapsto 1$. Then we have $t \cdot D\left(\Phi_{r}(t)\right)=\Phi_{r+1}(t)$. It is well known that $D$ satisfies the operator identity

$$
(t \cdot D)^{n}=\sum_{k=0}^{n} S(n, k) \cdot t^{k} \cdot D^{k}
$$

where the letters $S(n, k)$ denote the Stirling numbers of the second kind. (See, e.g., formula (34) of Section 6.6 on p. 218 of [16].) Using this formula for $D$ allows us to obtain

$$
\Phi_{i}(t)=\sum_{j=0}^{i} S(i, j) \cdot t^{j} \cdot D^{j}\left(\frac{1}{1-t}\right)=\sum_{j=0}^{i} S(i, j) \cdot t^{j} \cdot \frac{j !}{(1-t)^{j+1}}
$$

Assuming $S(0,0)=1$, this formula holds even for $i=0$. Thus (3) is equivalent to

$$
\mathscr{H}(K[\mathscr{C}], t)=1+\sum_{i=0}^{d} f_{i} \cdot t \cdot \sum_{j=0}^{i} S(i, j) \cdot t^{j} \cdot \frac{j !}{(1-t)^{j+1}}
$$

Introducing

$$
f_{j}^{\Delta} \stackrel{\text { def }}{=} \begin{cases}\sum_{i=j}^{d} f_{i} \cdot S(i, j) \cdot j ! & \text { when } 0 \leq j \leq d \\ 1 & \text { when } j=-1\end{cases}
$$

we may transform (4) into the following equivalent form:

$$
\mathscr{K}(K[\mathscr{E}], t)=\frac{\sum_{i=-1}^{d} f_{i}^{\Delta} \cdot t^{i+1} \cdot(1-t)^{d-i-1}}{(1-t)^{d}}
$$

\section{Initial Ideals and Triangulations}

In this section we describe the connection between the Stanley-Reisner ring of a triangulation of a cubical complex $\mathscr{E}$ via pulling the vertices, and the Stanley ring of this cubical complex, using the language and standard facts of Gröbner basis theory.

Let us first recall the definition of the Stanley-Reisner ring of a simplicial complex $\Delta$. (See, e.g., [21].)

Definition 8. Given a simplicial complex $\Delta$ with vertex set $V$, we define the Stanley-Reisner ring $K[\Delta]$ of $\Delta$ to be the factor ring $K\left[x_{v}: v \in V\right] / I(\Delta)$, where the ideal $I(\Delta)$ is generated by the $\operatorname{set}\left\{\mathbf{x}_{v_{1}} \cdots x_{v_{k}}: k \in \mathbb{N},\left\{v_{1}, \ldots, v_{k}\right\} \notin \Delta\right\}$. We call $I(\Delta)$ the face ideal of $\Delta$. 
Note that both $K[\mathscr{E}]$ and $K\left[\Delta_{<}(\mathscr{C})\right]$ are the factors of the same polynomial ring $K\left[x_{v}: v \in V\right]$. To express the connection between the face ideals $I\left(\Delta_{<}(\mathscr{C})\right)$ and $I(\mathscr{C})$, we need the following concepts of Gröbner basis theory.

Definition 9. Consider an arbitrary polynomial ring $K[X]$ over a field $K$. A monomial order on the set of monomials of $K[X]$ is a linear order $<$ on the semigroup of monomials such that if $m_{1}, m_{2}$, and $n$ are monomials, then

$$
m_{1}>m_{2} \text { implies } n \cdot m_{1}>n \cdot m_{2} \text {. }
$$

Given a monomial order $<$, for every polynomial $p \in K[X]$ we define the initial term init $<(p)$ of $p$ to be the largest term with respect to the term order $<$. Given an ideal $I$ of $K[X]$ we denote by init ${ }_{<}(I)$ the ideal generated by the initial terms of elements of $I$. A generating system $\left\{p_{1}, \ldots, p_{k}\right\}$ of $I$ is called a Gröbner basis with respect to the term order $<$, if init ${ }_{<}(I)$ is generated by the set \{init ${ }_{<}\left(p_{1}\right), \ldots$, init $\left.{ }_{<}\left(p_{k}\right)\right\}$.

In particular, we use reverse lexicographic term orders, which are defined as follows.

Definition 10. Let $K[X]$ be a polynomial ring and let $<$ be a linear order on the set of variables $X$. We define the reverse lexicographic order $<_{\text {rlex }}$ induced by $<$ as follows. Given two monomials $m$ and $n$, we write both of them in the form $m=x_{1}^{a_{1}} \cdots x_{k}^{a_{k}}, n=x_{1}^{b_{1}} \cdots x_{k}^{b_{k}}$ where $x_{1}>\cdots>x_{k}$. We set $m<_{\text {rlex }} n$ iff $\operatorname{deg}(m)$ $<\operatorname{deg}(n)$ holds or we have $\operatorname{deg}(m)=\operatorname{deg}(n)$ and $a_{i}>b_{i}$ for the last index $i$ with $a_{i} \neq b_{i}$.

Using the above definitions, the relation between $I(\mathscr{C})$ and $I\left(\Delta_{<}(\mathscr{C})\right)$ may be stated as follows.

Theorem 4. Let $\mathscr{C}$ be a cubical complex on the vertex set $V$ and let $<$ be any linear order on the vertices. Then we have the following identity:

$$
\text { init }_{<_{\text {lex }}}(I(\mathscr{E}))=I\left(\Delta_{<}(\mathscr{E})\right) \text {. }
$$

In words, the initial ideal of the face ideal of $\mathscr{E}$ with respect to the reverse lexicographic order induced by $<$ is the face ideal of the triangulation of $\mathscr{C}$ via pulling the vertices with respect to the order $<$.

Proof. By Definition 6 and Corollary 1, the face ideal $I(\mathscr{C})$ is a binomial ideal, i.e., it has a generating system consisting only of binomials. (A binomial is a linear combination of at most two monomials.) Moreover, all monomials of $I(\mathscr{C})$ belong to $I_{1}(\mathscr{E})$. It is well known in the theory of binomial ideals, that every binomial ideal with respect to any term order has a reduced Gröbner basis consisting only of binomials. (See, e.g., Proposition 1 of [8].) This is true, because if we start Buch- 
berger's algorithm (described, e.g., in Section 5.5 [3]) to compute a Gröbner basis on a set of binomials, every newly added polynomial will be a binomial. In our case, we have a reduced Gröbner basis $\mathscr{G}=\mathscr{G}_{1} \cup \mathscr{G}_{2}$, where $\mathscr{G}_{1}$ is the set of monomials minimally generating $I_{1}(\mathscr{C})$, and $\mathscr{I}_{2}$ is the reduced Gröbner basis with respect to $<_{\text {rlex }}$ of $I_{2}(\mathscr{C})$. It is easy to verify that the elements of $\mathscr{G}_{2}$ will be differences of monomials of the form $x_{u_{1}} \cdots x_{u_{k}}-x_{v_{1}} \cdots x_{v_{k}}$, where $\operatorname{Cspan}\left(\left\{u_{1}, \ldots, u_{k}\right\}\right)$ exists and the multisets $\left\lfloor u_{1}, \ldots, u_{k}\right\rfloor$ and $\left[v_{1}, \ldots, v_{k}\right\rfloor$ are equivalent. Moreover, a monomial $x_{u_{1}} \cdots x_{u_{k}} \notin I_{1}(\mathscr{E})$ is not the leading term of a binomial $x_{u_{1}} \cdots x_{u_{k}}-x_{v_{1}} \cdots x_{v_{k}} \in$ $I_{2}(\mathscr{C})$ if and only if the set $\left\{u_{1}, \ldots, u_{k}\right\}$ is a face of $\Delta_{<}(\mathscr{E})$. In fact $x_{u_{1}} \cdots x_{u_{k}} \notin I_{1}(\mathscr{E})$ holds iff $\left\{u_{1}, \ldots, u_{k}\right\}$ is contained in some face $\sigma \in \mathscr{E}$. Assuming $u_{1} \geq \cdots \geq u_{k}$, there is a multiset $\left\lfloor v_{1}, \ldots, v_{k}\right\rfloor$ equivalent to $\left\lfloor u_{1}, \ldots, u_{k}\right\rfloor$ with $x_{v_{1}} \cdots x_{v_{k}}<_{\text {rlex }} x_{u_{1}}$ $\cdots x_{u_{k}}$ iff for some $i \in\{2, \ldots, k\}$ we have $u_{i} \neq \delta_{<}\left(\operatorname{Cspan}\left(u_{1}, \ldots, u_{i-1}\right)\right)$. (This is a consequence of Lemma 2.) Thus init $<_{\text {rlex }}(I(\mathscr{C}))$ is generated by square-free monomials, and these square-free monomials are the same as those generating $I\left(\Delta_{<}(\mathscr{C})\right)$.

Corollary 3. For any cubical complex $\mathscr{C}$, and any of its triangulations via pulling the vertices $\Delta_{<}(\mathscr{C})$ we have

$$
\mathscr{H}(K[\mathscr{C}], t)=\mathscr{H}\left(K\left[\Delta_{<}(\mathscr{C})\right], t\right)
$$

Proof. It is well known in the theory of Gröbner bases that for every polynomial ring $K[X]$, every ideal $I$ of this polynomial ring, and every term order $\prec$ the Hilbert-series of $K[X] / I$ is equal to the Hilbert series of $K[X] /$ init ${ }_{\prec}(I)$. (See, e.g., Lemma 9.26 and Proposition 6.52 of [3].) Thus we have

$$
\mathscr{H}(K[\mathscr{C}], t)=\mathscr{H}\left(K\left[x_{v}: v \in V\right] / \text { init }_{<_{\text {tlex }}}(I(\mathscr{C})), t\right)
$$

and the statement follows from Theorem 4.

Corollary 4. For any d-dimensional cubical complex $\mathscr{C}$, and any order $<$ on its vertices, the vector $\left(f_{-1}^{\Delta}, \ldots, f_{d}^{\Delta}\right)$ given by (5) is the f-vector of the simplicial complex $\Delta_{<}(\mathscr{C})$.

Proof. This is a straight consequence of (6), Corollary 3, and [21, Chapter II, Section 1, Theorem 1.4].

Remarks. 1. The fact that a square-free initial ideal is the face ideal of a simplicial complex is widely used, see, e.g., Section 6 of [22] or the Introduction of [10].

2. The above proof was inspired by (and is much simpler than) the analogous result of Sturmfels for initial ideals of toric ideals in [22]. The special case when the cubical complex is a standard $n$-cube is implicit in Corollary 5.2 of [22]. 
3. As a special case of Corollary 4 , we obtain that every triangulation via pulling the vertices of a standard $n$-cube has the same $f$-vector. This could also be deduced from Corollary 2.7 of [20], because using Theorem 2.3 of [20] it is easy to show that the standard $n$-cube is a compressed polytope.

\section{Shellable Cubical Complexes}

In the definition of shellable cubical complexes we need the notions of ball and sphere. Remember that an $n$-cube $\mathscr{C}^{n}$ has a standard geometric realization $\phi: V\left(\mathscr{E}^{n}\right) \rightarrow \mathbb{R}^{n}$, where $\phi$ is a bijection between $V\left(\mathscr{C}^{n}\right)$ and $\{0,1\}^{n}$, such that vertices connected by an edge go into the vertices of $[0,1]^{n}$ connected by an edge. (See Definition 3.)

Definition 11. A collection $\left\{F_{1}, F_{2}, \ldots, F_{k}\right\}$ of facets of the boundary of an $n$-cube is called an $(n-1)$-dimensional ball or $(n-1)$-dimensional sphere, respectively, if the set $\cup_{i=1}^{k} \operatorname{conv}\left(\phi\left(F_{i}\right)\right)$ is homeomorphic to an $(n-1)$-dimensional ball or sphere, respectively.

As in [15], we encode the nonempty faces of $\mathscr{C}^{n}$ with vectors $\left(u_{1}, u_{2}, \ldots, u_{n}\right) \in$ $\{0,1, *\}^{n}$ in the following way. Consider a standard geometric realization $\phi: \mathscr{C}^{n} \rightarrow \mathbb{R}^{n}$ For a nonempty face $\sigma \in \mathscr{C}^{n}$ and $i \in\{1,2, \ldots, n\}$ set $u_{i}=0$ or 1 , respectively, if the $i$ th coordinate of every element of $\phi(\sigma)$ is 0 or 1 , respectively. Otherwise we set $u_{i}=*$. Using this coding, the facets of $\mathscr{C}^{n}$ correspond to the vectors $\left(u_{1}, \ldots, u_{n}\right)$ for which exactly one of the $u_{i}$ 's is not a $*$-sign.

Definition 12. Let $A_{i}^{0}$ (resp. $\left.A_{i}^{1}\right)$ stand for the facet $\left(u_{1}, u_{2}, \ldots, u_{n}\right)$ with $u_{i}=0$ (resp. $u_{i}=1$ ) and $u_{j}=*$ for $j \neq i$. Let $\left\{F_{1}, \ldots, F_{k}\right\}$ be a collection of facets of $\partial\left(\mathscr{E}^{n}\right)$. Let $r$ be the number of $i$ 's such that exactly one of $A_{i}^{0}$ and $A_{i}^{1}$ belong to $\left\{F_{1}, \ldots, F_{k}\right\}$, and let $s$ be the number of $i$ 's such that both $A_{i}^{0}$ and $A_{i}^{1}$ belong to $\left\{F_{1}, \ldots, F_{k}\right\}$. We call $(r, s)$ the type of $\left\{F_{1}, \ldots, F_{k}\right\}$.

Note that when the type of $\left\{F_{1}, \ldots, F_{k}\right\}$ is $(r, s)$ then there are exactly $n-r-s$ coordinates $i$ such that neither $A_{i}^{0}$ nor $A_{i}^{1}$ belong to $\left\{F_{1}, \ldots, F_{k}\right\}$.

The following observation, originally due to Adin [1], gives a full description of those collections of facets $\left\{F_{1}, \ldots, F_{k}\right\}$ which are an $(n-1)$-dimensional ball or sphere.

Lemma 3. The collection of facets $\left\{F_{1}, \ldots, F_{k}\right\}$ of the boundary of an $n$-cube is an $(n-1)$-sphere if and only if it has type $(0, n)$ and it is an $(n-1)$-ball if and only if its type $(r, s)$ satisfies $r>0$.

Remark. Lemma 3 allows us to define $(n-1)$-balls or $(n-1)$-spheres combinatorially, by prescribing their types. 
Definition 13. A cubical complex $\mathscr{C}$ is pure if all facets of $\mathscr{C}$ have the same dimension. We define shellable cubical complexes as follows:

1. The empty set is a $((-1)$-dimensional) shellable cubical complex.

2. A point is a (zero-dimensional) shellable complex.

3. A $d$-dimensional pure complex $\mathscr{C}$ is shellable if its facets can be listed in a linear order $F_{0}, F_{1}, \ldots, F_{n}$ such that for each $k \in\{1,2, \ldots, n\}$ the subcomplex $\left.\mathscr{C}\right|_{F_{k}} \cap\left(\left.\left.\mathscr{E}\right|_{F_{1}} \cup \cdots \cup \mathscr{C}\right|_{F_{k-1}}\right)$ is a pure complex of dimension $(d-1)$ such that its maximal dimensional faces form a $(d-1)$-dimensional ball or sphere.

By abuse of notation we say that the attachment of $\left.\mathscr{C}\right|_{F_{k}}$ to $\left.\left.\mathscr{E}\right|_{F_{1}} \cup \cdots \cup \mathscr{C}\right|_{F_{k-1}}$ in a shelling $F_{0}, F_{1}, \ldots, F_{k}$ has type $(r, s)$ if the set of facets of $\left.\mathscr{C}\right|_{F_{k}} \cap\left(\left.\mathscr{E}\right|_{F_{1}} \cup \cdots \cup\right.$ $\left.\left.\mathscr{C}\right|_{F_{k}-1}\right)$ considered as a collection of facets of $\left.\mathscr{C}\right|_{F_{k}}$ has type $(r, s)$.

The definition of shellability often allows us to prove properties of shellable cubical complexes by induction on shelling, i.e., by induction on their dimension and the number of their facets. One of the most important results obtainable this way is the following theorem.

Theorem 5. If $\mathscr{C}$ is a shellable cubical complex, then the Stanley ring $K[\mathscr{E}]$ is $a$ Cohen-Macaulay ring.

Proof (Sketch). The basic idea of the proof is due to Hochster. We observe that if $I_{1}$ and $I_{2}$ are perfect ideals of the same dimension, $k$, and $I_{1}+I_{2}$ is a perfect ideal of dimension $k-1$, then $I_{1} \cap I_{2}$ is a perfect ideal. (This is item (ii) in the proof of Theorem $2^{\circ}$ of [12], essentially equivalent to Proposition 18 of [13].) Hence, like in the proof of Theorem $2^{\circ}$ of [12], we can prove the perfectness of the face ideal $I(\mathscr{C})$ of a shellable cubical complex $\mathscr{E}$ by induction on the number of facets. We leave the details to the reader. (Note the well-known fact that the perfectness of an ideal in a polynomial ring implies the Cohen-Macaulay property of the factor by this ideal-see, e.g., Theorem 3.5 .8 of [2].)

Another example to the use of induction on shelling is the following elementary lemma, which we use later.

Lemma 4. The edge-graph of a shellable cubical complex of dimension at least two is bipartite.

Proof. We use induction on the number of facets. Let $F_{1}, \ldots, F_{k}$ be a shelling of $\mathscr{C}$. By the induction hypothesis, the complex $\left.\left.\mathscr{C}\right|_{F_{1}} \cup \cdots \cup \mathscr{C}\right|_{F_{k-1}}$ has a bipartite edge-graph. Clearly, $\left.\mathscr{C}\right|_{F_{k}}$ has a bipartite edge-graph: when we represent its vertices, as vertices of the standard $d$-cube $[0,1]^{d}$, an appropriate coloring with two colors is to color the vertices according to the parity of the sum of their coordinates. It is easy to check that the edge-graph of $\left.\mathscr{C}\right|_{F_{k}} \cap\left(\left.\left.\mathscr{C}\right|_{F_{1}} \cup \cdots \cup \mathscr{C}\right|_{F_{k-1}}\right)$ is a connected graph. Thus the induction step follows from the fact that essentially there is only one way to color a connected bipartite graph. 


\section{A Homogeneous Generating System of Degree Two for $I(\mathscr{C})$}

Theorem 2 inspires the following question. Let $I_{1}^{\prime \prime}(\mathscr{E})$ be the ideal generated by the monomials $x_{u} \cdot x_{v}$ such that the pair $\{u, v\} \subseteq V$ is not contained in any face. When do we have $I(\mathscr{C})=I_{1}^{\prime \prime}(\mathscr{C})+I_{2}(\mathscr{E})$ ? For such complexes $I(\mathscr{C})$ is generated by homogeneous forms of degree two.

Definition 14. We call a cubical complex well behaved when it satisfies $I(\mathscr{C})=$ $I_{1}^{\prime \prime}(\mathscr{C})+I_{2}(\mathscr{E})$.

The following lemma is a straightforward consequence of Theorem 2 and of the trivial inclusion $I_{1}^{\prime \prime}(\mathscr{C}) \subseteq I_{1}^{\prime}(\mathscr{C})$.

Lemma 5. A cubical complex is well behaved iff for every triple $\left\lfloor u_{1}, u_{2}, u_{3}\right\rfloor$ either $\left\{u_{1}, u_{2}, u_{3}\right\}$ is contained in a face of $\mathscr{E}$ or there is a $\left\lfloor v_{1}, v_{2}, v_{3}\right\rfloor$ equivalent to $\left\lfloor u_{1}, u_{2}, u_{3}\right\rfloor$ such that $\left\{v_{1}, v_{2}\right\}$ is not contained in any face of $\mathscr{E}$.

We use the statement of Lemma 5 as an equivalent definition of well-behaved cubical complexes.

Example. Figure 1 represents a not well-behaved cubical complex. The facets of the complex are $A B E D, B C E F$, and $A C D F$.

It is easy to verify that, for the triple $\lfloor A, C, E\rfloor$ and for any equivalent triple, any two elements of the triple are contained in a face, but there is no face containing all three of them.

Conjecture 1. Every shellable cubical complex is well behaved.

The following lemmas are statements about the properties of an eventual minimal counterexample to Conjecture 1. (Minimality always means minimality of the number of facets.) At the end we do not get a proof of the conjecture, but the properties shown allow us to exclude all shellable subcomplexes of a boundary complex of a convex cubical polytope from the class of shellable not well-behaved cubical complexes.

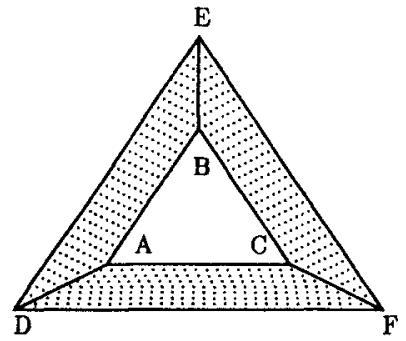

Fig. 1. A not well-behaved cubical complex. 
Definition 15. Let $\mathscr{C}$ be a not well-behaved shellable cubical complex. We call the triple $\left\lfloor u_{1}, u_{2}, u_{3}\right\rfloor$ a counterevidence if any two of $u_{1}, u_{2}$, and $u_{3}$ are contained in some face of $\mathscr{E}$, but no face contains the set $\left\{u_{1}, u_{2}, u_{3}\right\}$, and the same holds for all equivalent triples in $\mathscr{C}$.

Clearly, $\mathscr{E}$ is a counterexample to Conjecture 1 exactly when $\mathscr{E}$ contains a counterevidence. The following lemma tells us how a counterevidence must lie in a minimal counterexample.

Lemma 6. Let $\mathscr{C}$ be a minimal not well-behaved shellable cubical complex and let $F_{1}, F_{2}, \ldots, F_{k}$ be a shelling of $\mathscr{C}$. Then every counterevidence $\left\lfloor u_{1}, u_{2}, u_{3}\right\rfloor$ has exactly one element outside $F_{k}$, and two elements in $F_{k}$.

Proof. The triple $\left\lfloor u_{1}, u_{2}, u_{3}\right\rfloor$ cannot be a counterevidence, if all three $u_{i}$ 's lie in $F_{k}$. Thus at least one of them must lay outside $F_{k}$. It at least two of $u_{1}, u_{2}, u_{3}$ are not in $F_{k}$, then any face containing at least two of them is also contained in $\left.\mathscr{E}\right|_{F_{1}} \cup \cdots \cup$ $\left.\mathscr{C}\right|_{F_{k-1}}$. In particular, we must have $\left\{u_{1}, u_{2}, u_{3}\right\} \subset F_{1} \cup \cdots \cup F_{k-1}$. By minimality, $\left\lfloor u_{1}, u_{2}, u_{3}\right\rfloor$ is not a counterevidence in the shellable complex $\left.\left.\mathscr{C}\right|_{F_{1}} \cup \cdots \cup \mathscr{C}\right|_{F_{k-1}}$. Thus there is an equivalent $\left\lfloor v_{1}, v_{2}, v_{3}\right\rfloor$ such that $\left\{v_{1}, v_{2}, v_{3}\right\}$ is contained in some face of $\left.\left.\mathscr{E}\right|_{F_{1}} \cup \cdots \cup \mathscr{C}\right|_{F_{k-1}}$. However, then the same holds in $\mathscr{E}$ and so $\left\lfloor u_{1}, u_{2}, u_{3}\right\rfloor$ is not a counterevidence. Hence the only way for $\left\lfloor u_{1}, u_{2}, u_{3}\right\rfloor$ to be a counterevidence is to contain exactly one element outside $F_{k}$.

Corollary 5. Let $\mathscr{C}$ be a minimal not well-behaved shellable cubical complex, let $F_{1}, F_{2}, \ldots, F_{k}$ be a shelling of $\mathscr{C}$, and let $\left\lfloor u_{1}, u_{2}, u_{3}\right\rfloor$ be a counterevidence such that $u_{1}, u_{2} \in F_{k}$ and $u_{3} \notin F_{k}$. Then, for every $u \in \operatorname{Cspan}\left(\left\{u_{1}, u_{2}\right\}\right)$, the face $\operatorname{Cspan}\left(\left\{u_{3}, u\right\}\right)$ has exactly half of its vertices in $F_{k}$.

Proof. If necessary, we can replace the diagonal $\left\lfloor u_{1}, u_{2}\right\rfloor$ by another diagonal of Cspan $\left(\left\{u_{1}, u_{2}\right\}\right)$ such that $u=u_{1}$ holds, and so $\operatorname{Cspan}\left(\left\{u_{3}, u\right\}\right)$ exists, and we may assume $u_{1}=u$. By $u_{3} \notin F_{k}$, at most half of $\operatorname{Cspan}\left(\left\{u_{3}, u\right\}\right)$ belongs to $F_{k}$. If less than half is contained in $F_{k}$, then the diagonal $\left\lfloor u_{1}, u_{3}\right\rfloor$ may be replaced by a diagonal $\left\lfloor u_{1}^{\prime}, u_{3}^{\prime}\right\rfloor$ such that both $u_{1}^{\prime}$ and $u_{3}^{\prime}$ are outside $F_{k}$. Thus the triple $\left\lfloor u_{1}^{\prime}, u_{2}, u_{3}^{\prime}\right\rfloor$ (which is equivalent to $\left\lfloor u_{1}, u_{2}, u_{3}\right\rfloor$ ) will not be a counterevidence by Lemma 6 .

Proposition 1. Assume that, for the shelling $F_{1}, \ldots, F_{k}$ of $\mathscr{E}$, the attachment of $\left.\mathscr{C}\right|_{F_{k}}$ to $\left.\bigcup_{i=1}^{k-1} \mathscr{C}\right|_{F_{i}}$ has type $(r, 0)$. Then $\mathscr{C}$ cannot be a minimal not well-behaved shellable complex.

Proof. Assume the contrary. When the type is $(r, 0)$ then $F_{k} \backslash\left(F_{1} \cup \cdots \cup F_{k-1}\right)$ is not empty: there is at least one vertex which was added when we added $F_{k}$. Let $\left\lfloor u_{1}, u_{2}, u_{3}\right\rfloor$ be a counterexample with $u_{1}, u_{2} \in F_{k}, u_{3} \notin F_{k}$. If $\operatorname{Cspan}\left(\left\{u_{1}, u_{2}\right\}\right)$ contains a newly added vertex, then-after replacing eventually $u_{1}$ and $u_{2}$ with another 
diagonal of $\operatorname{Cspan}\left(\left\{u_{1}, u_{2}\right\}\right)$-we may assume $u_{2} \in F_{k} \backslash\left(F_{1} \cup \cdots \cup F_{k-1}\right)$. However then $\left\{u_{2}, u_{3}\right\}$ is not contained in any face of $\mathscr{C}$, and we get a contradiction. Thus we must have $\operatorname{Cspan}\left(\left\{u_{1}, u_{2}\right\}\right) \subset F_{1} \cup \cdots \cup F_{k-1}$. In this case, however, any face containing at least two of $u_{1}, u_{2}$, and $u_{3}$ is contained in $\left.\left.\mathscr{E}\right|_{F_{1}} \cup \cdots \cup \mathscr{C}\right|_{F_{k-1}}$ and so $\left\lfloor u_{1}, u_{2}, u_{3}\right\rfloor$ is a counterevidence in this smaller complex already.

Lemma 7. Let $\mathscr{C}$ be a minimal not well-behaved shellable cubical complex and let $F_{1}, F_{2}, \ldots, F_{k}$ be a shelling of $\mathscr{C}$. Assume that there is a pair $H, H^{\prime}$ of subfacets which are opposite facets of $\partial\left(\left.\mathscr{C}\right|_{F_{k}}\right)$ and both belong to $\left.\left.\mathscr{C}\right|_{F_{1}} \cup \cdots \cup \mathscr{C}\right|_{F_{k-1}}$. Let $\left\lfloor u_{1}, u_{2}, u_{3}\right\rfloor$ be a counterevidence such that $u_{1}, u_{2} \in F_{k}, u_{3} \notin F_{k}$. Then there is a face of $\mathscr{E}$ containing $\operatorname{Cspan}\left(\left\{u_{1}, u_{2}\right\}\right) \cap H$ and $u_{3}$.

Proof. If $\operatorname{Cspan}\left(\left\{u_{1}, u_{2}\right\}\right) \cap H=\varnothing$, then we have $\left.\operatorname{Cspan}\left(\left\{u_{1}, u_{2}\right\}\right) \subseteq H^{\prime} \in \mathscr{C}\right|_{F_{1}}$ $\left.\cup \cdots \cup \mathscr{E}\right|_{F_{k-1}}$, meaning that $\left.\left.\mathscr{C}\right|_{F_{1}} \cup \cdots \cup \mathscr{E}\right|_{F_{k-1}}$ was already a counterexample. Similar contradiction with minimality arises when we assume $\operatorname{Cspan}\left(\left\{u_{1}, u_{2}\right\}\right) \cap H^{\prime}$ $=\varnothing$. Thus we may suppose $u_{1} \notin H$ and $u_{2} \in H$, and we may renumber all equivalent triples $\left\lfloor v_{1}, v_{2}, v_{3}\right\rfloor$ such that $v_{3} \notin F_{k}, v_{1} \in H^{\prime}$, and $v_{2} \in H$ hold. Let $u_{1}^{\prime}$ be the projection of $u_{1}$ onto $H$. Then we have $\operatorname{Cspan}\left(\left\{u_{1}^{\prime}, u_{2}\right\}\right)=\operatorname{Cspan}\left(\left\{u_{1}, u_{2}\right\}\right) \cap H$. If the set $\left\{u_{1}^{\prime}, u_{2}, u_{3}\right\}$ is contained in a face, then we are done. Otherwise, given the fact that the cubical span of any two of $u_{1}^{\prime}, u_{2}$, and $u_{3}$ is contained in $\left.\mathscr{C}\right|_{F_{1}} \cup \cdots \cup$ $\left.\mathscr{C}\right|_{F_{k-1}}$, a well-behaved shellable cubical complex, we obtain that the triple $\left\lfloor u_{1}^{\prime}, u_{2}, u_{3}\right\rfloor$ is equivalent to a triple $\left\lfloor z_{1}, z_{2}, z_{3}\right\rfloor$ such that there is no face of $\left.\left.\mathscr{C}\right|_{F_{1}} \cup \cdots \cup \mathscr{C}\right|_{F_{k-1}}$ containing $\left\{z_{1}, z_{2}\right\}$. Consider a sequence of replacing diagonals, which demonstrates the equivalence of $\left\lfloor u_{1}^{\prime}, u_{2}, u_{3}\right\rfloor$ and $\left\lfloor z_{1}, z_{2}, z_{3}\right\rfloor$. Assume that $\left\lfloor u_{1}, u_{2}, u_{3}\right\rfloor$ was chosen from its equivalence class such that this derivation of equivalence is the shortest possible.

If the first step is replacing the triple $\left\{u_{1}^{\prime}, u_{2}, u_{3}\right\rfloor$ with $\left\lfloor w_{1}^{\prime}, w_{2}, u_{3}\right\rfloor$ where $\left\{w_{1}^{\prime}, w_{2}\right\}$ is a diagonal of $\operatorname{Cspan}\left(\left\{u_{1}^{\prime}, u_{2}\right\}\right)$, then we get a contradiction with the minimality of the derivation. In fact, let $w_{1}$ be the projection of $w_{1}^{\prime}$ onto $H^{\prime}$. It is easy to check that $\left\{w_{1}, w_{2}\right\}$ is a diagonal of $\operatorname{Cspan}\left(\left\{u_{1}, u_{2}\right\}\right)$, so $\left\lfloor w_{1}, w_{2}, u_{3}\right\rfloor$ is equivalent to $\left\lfloor u_{1}, u_{2}, u_{3}\right\rfloor$, and there is a shorter derivation of equivalence between $\left\lfloor w_{1}^{\prime}, w_{2}, u_{3}\right\rfloor$ and $\left\lfloor z_{1}, z_{2}, z_{3}\right\rfloor$. We also get a contradiction when we assume that our first step was to replace $\left\lfloor u_{1}^{\prime}, u_{2}, u_{3}\right\rfloor$ with $\left\lfloor u_{1}^{\prime}, w_{2}, w_{3}\right\rfloor$, where $\left\{w_{2}, w_{3}\right\}$ is a diagonal of $\operatorname{Cspan}\left(\left\{u_{2}, u_{3}\right\}\right)$. In this case the very same replacement can be performed on $\left\lfloor u_{1}, u_{2}, u_{3}\right\rfloor$ and we obtain the equivalent triple $\left\lfloor u_{1}, w_{2}, w_{3}\right\rfloor$, from which we have a shorter derivation.

Hence we are left with the case when the first step of the derivation involves replacing $\left\lfloor u_{1}^{\prime}, u_{2}, u_{3}\right\rfloor$ with $\left\lfloor w_{1}^{\prime}, u_{2}, w_{3}\right\rfloor$, where $\left\{w_{1}^{\prime}, w_{3}\right\}$ is a diagonal of $\operatorname{Cspan}\left(\left\{u_{1}^{\prime}, u_{3}\right\}\right)$ such that $w_{1}^{\prime} \in H$ and $w_{3} \notin F_{k}$. Let $q_{1}$ be the projection of $u_{2}$ onto $H^{\prime}$. Then $\left\lfloor q_{1}, w_{1}^{\prime}, w_{3}\right\rfloor$ is equivalent to $\left\lfloor u_{1}, u_{2}, u_{3}\right\rfloor$, so the first step is again unnecessary.

Therefore we may assume that at least two of $u_{1}^{\prime}, u_{2}$, and $u_{3}$ are not contained in a common face. This pair cannot be $\left\{u_{1}^{\prime}, u_{2}\right\} \subseteq F_{k}$ and it cannot be $\left\{u_{2}, u_{3}\right\}$ because then $\left\{u_{1}, u_{2}, u_{3}\right\rfloor$ is not a counterexample. Finally, if $\left\{u_{1}^{\prime}, u_{3}\right\}$ is not contained in any 
face, then we obtain a contradiction since $\left\lfloor q_{1}, u_{1}^{\prime}, u_{3}\right\rfloor$ is not a counterevidence but is equivalent to $\left\lfloor u_{1}, u_{2}, u_{3}\right\rfloor$.

Lemma 8. Assume $F_{1}, \ldots, F_{k}$ is a shelling of a minimal not well-behaved complex $\mathscr{C}$, and $\left\lfloor u_{1}, u_{2}, u_{3}\right\rfloor$ is a counterevidence with $u_{3} \notin F_{k}$. Assume $H$ and $H^{\prime}$ are opposite facets of $\partial\left(\left.\mathscr{E}\right|_{F_{k}}\right)$, such that they both belong to $\left.\left.\mathscr{C}\right|_{F_{1}} \cup \cdots \cup \mathscr{C}\right|_{F_{k-1}}$. Then there is no edge $\{v, w\} \in \operatorname{Cspan}\left(\left\{u_{1}, u_{2}\right\}\right)$ for which $v \in H, w \in H^{\prime}$ would hold and Cspan $\left(\left\{u_{3}, v, w\right\}\right)$ would exist.

Proof. Assume the contrary. Since $\{v, w\}$ is an edge, either $\left\{u_{3}, v\right\}$ or $\left\{u_{3}, w\right\}$ is a diagonal of Cspan $\left(\left\{u_{3}, v, w\right\}\right)$. Without loss of generality we may assume that $\left\{u_{3}, v\right\}$ is a diagonal. We also may assume that the triple $\left\lfloor u_{1}, u_{2}, u_{3}\right\rfloor$ was chosen in such a way that $u_{1}=v$ holds. (If not, we can replace the pair $\left\lfloor u_{1}, u_{2}\right\rfloor$ with another pair containing $v \in C \operatorname{span}\left(\left\{u_{1}, u_{2}\right\}\right)$.) Let $u_{3}^{\prime}$ be the vertex diagonally opposite to $w$ in $\operatorname{Cspan}\left(\left\{u_{3}, v, w\right\}\right)$. Then $\left\lfloor u_{1}, u_{2}, u_{3}\right\rfloor$ is equivalent to $\left\lfloor w, u_{2}, u_{3}^{\prime}\right\rfloor$ and here we have $u_{3}^{\prime} \notin F_{k}$ and $\left.\left.\left\{w, u_{2}\right\} \subseteq H^{\prime} \in \mathscr{C}\right|_{F_{1}} \cup \cdots \cup \mathscr{C}\right|_{F_{k-1}}$, contradicting the assumption about the minimality of $\mathscr{C}$.

Proposition 2. If $\mathscr{C}$ has a shelling $F_{1}, \ldots, F_{k}$ such that the attachment of $\left.\mathscr{E}\right|_{F_{k}}$ to $\left.\bigcup_{i=1}^{k-1} \mathscr{E}\right|_{F_{i}}$ has type $(r, s)$ with $s \geq 2$, then $\mathscr{E}$ is not a minimal not well-behaved shellable complex.

Proof. Assume the contrary. Let $H_{1}, H_{1}^{\prime}$ and $H_{2}, H_{2}^{\prime}$ be pairs of subfacets which are opposite in $F_{k}$, all belonging to $\left.\mathscr{C}\right|_{F_{k}} \cap\left(\left.\left.\mathscr{C}\right|_{F_{1}} \cup \cdots \cup \mathscr{C}\right|_{F_{k-1}}\right)$. Assume furthermore that $\left\lfloor u_{1}, u_{2}, u_{3}\right\rfloor$ is a counterevidence satisfying $u_{1}, u_{2} \in F_{k}, u_{3} \notin F_{k}$. Then $\operatorname{Cspan}\left(\left\{u_{1}, u_{2}\right\}\right) \cap H_{i}$ and $\operatorname{Cspan}\left(\left\{u_{1}, u_{2}\right\}\right) \cap H_{i}^{\prime}$ are nonempty by the minimality of $\mathscr{C}$. By Lemma $7, \operatorname{Cspan}\left(\left\{u_{1}, u_{2}\right\}\right) \cap H_{1}$ is contained in a face with $u_{3}$. However, then we can find $v, w \in \operatorname{Cspan}\left(\left\{u_{1}, u_{2}\right\}\right) \cap H_{1}$ such that $\{v, w\}$ is an edge and we have $v \in H_{2}, w \in H_{2}^{\prime}$, contradicting Lemma 8 .

Lemma 9. Let $\mathscr{E}$ be a minimal not well-behaved shellable complex. Let $H$ and $H^{\prime}$ be opposite facets of $\partial\left(\left.\mathscr{C}\right|_{F_{k}}\right)$ such that $H$ also belongs to $\left(\left.\left.\mathscr{C}\right|_{F_{1}} \cup \cdots \cup \mathscr{C}\right|_{F_{k-1}}\right)$ but $H^{\prime}$ does not. Assume, there is a counterevidence $\left\lfloor u_{1}, u_{2}, u_{3}\right\rfloor$, such that $u_{1}, u_{2} \in F_{k}$, $u_{3} \notin F_{k}$, and $\operatorname{Cspan}\left(\left\{u_{1}, u_{2}\right\}\right) \cap H \neq \varnothing$ hold. Then for any $u \in \operatorname{Cspan}\left(\left\{u_{1}, u_{2}\right\}\right) \cap H^{\prime}$ we have

$$
\operatorname{Cspan}\left\{\left(u_{3}, u\right)\right\} \cap F_{k} \subseteq H^{\prime}
$$

Proof. Note first that $\operatorname{Cspan}\left(\left\{u_{1}, u_{2}\right\}\right) \cap H^{\prime} \neq \varnothing$ otherwise $\left\lfloor u_{1}, u_{2}, u_{3}\right\rfloor$ would also be a counterevidence in $\left(\left.\left.\mathscr{C}\right|_{F_{1}} \cup \cdots \cup \mathscr{C}\right|_{F_{k-1}}\right)$. Without loss of generality we may assume $u=u_{1}$ and so $u_{2} \in H$. By Corollary 5 the face Cspan $\left(\left\{u_{3}, u_{1}\right\}\right)$ has exactly half of its vertices in $F_{k}$. In particular, $u_{3}$ is connected by an edge to a unique vertex 
$v \in F_{k}$ and we have $\operatorname{Cspan}\left(\left\{u_{3}, u_{1}\right\}\right) \cap F_{k}=\operatorname{Cspan}\left(\left\{u_{1}, v\right\}\right)$. Thus we only need to show $v \in H^{\prime}$. If not, then we can replace the diagonal $\left\lfloor u_{3}, u_{1}\right\rfloor$ with a diagonal $\left\lfloor u_{3}^{\prime}, v\right\rfloor$ and obtain an equivalent triple $\left\lfloor v, u_{2}, u_{3}^{\prime}\right\rfloor$ with $v, u_{2} \in H$, contradicting the assumption of minimality of $\mathscr{C}$.

Proposition 3. Let $\mathscr{C}$ be shellable d-dimensional minimal not well-behaved cubica! complex, with shelling $F_{1}, F_{2}, \ldots, F_{k}$. Then the type of the attachment of $\left.\mathscr{C}\right|_{F_{k}}$ to $\left.\left.\mathscr{E}\right|_{F_{1}} \cup \cdots \cup \mathscr{C}\right|_{F_{k-1}}$ cannot be $(r, d-r)$.

Proof. Assume the contrary. By Propositions 1 and 2 we may assume that the type of the attachment of $\left.\mathscr{E}\right|_{F_{k}}$ to $\left.\left.\mathscr{E}\right|_{F_{1}} \cup \cdots \cup \mathscr{E}\right|_{F_{k-1}}$ is $(d-1,1)$. Thus, taking a standard geometric realization $\phi$ of $\left.\mathscr{C}\right|_{F_{k}}$, we may assume that exactly the following facets of $\partial\left(\left.\mathscr{C}\right|_{F_{k}}\right)$ belong to $\left.\left.\mathscr{C}\right|_{F_{1}} \cup \cdots \cup \mathscr{C}\right|_{F_{k-1}}: A_{1}^{0}, A_{2}^{0}, \ldots, A_{d-1}^{0}, A_{d}^{0}$, and $A_{d}^{1}$.

Let $\left\lfloor u_{1}, u_{2}, u_{3}\right\rfloor$ be a counterevidence such that $u_{1}, u_{2} \in F_{k}, u_{3} \notin F_{k}$, and $\operatorname{dim} \operatorname{Cspan}\left(\left\{u_{1}, u_{2}\right\}\right)$ is maximal under these conditions. Then, similarly to Corollary 5 , we can show that for every $u \in \operatorname{Cspan}\left(\left\{u_{1}, u_{2}\right\}\right)$ the face $\operatorname{Cspan}\left(\left\{u_{3}, u\right\}\right)$ exists and has exactly half of its vertices in $\operatorname{Cspan}\left(\left\{u_{1}, u_{2}\right\}\right)$. In fact, without loss of generality we may assume $u_{1}=u$ and so $\operatorname{Cspan}\left(\left\{u_{3}, u\right\}\right)$ exist. At most half of $\operatorname{Cspan}\left(\left\{u_{3}, u_{1}\right\}\right)$ may belong to Cspan $\left(\left\{u_{1}, u_{2}\right\}\right)$, because otherwise $F_{k}$ would also contain more than half of the vertices of $\operatorname{Cspan}\left(\left\{u_{3}, u_{1}\right\}\right)$, in contradiction with Corollary 5 . If less than half of the vertices of $\operatorname{Cspan}\left(\left\{u_{3}, u_{1}\right\}\right)$ belong to $C \operatorname{span}\left(\left\{u_{1}, u_{2}\right\}\right)$, then there is a $u_{1}^{\prime} \in$ $\operatorname{Cspan}\left(\left\{u_{3}, u_{1}\right\}\right)$ such that $u_{1}^{\prime}$ is connected to $u_{1}$ by an edge, and $u_{1}^{\prime} \in F_{k} \backslash$ $\operatorname{Cspan}\left(\left\{u_{1}, u_{2}\right\}\right)$. Let $u_{3}^{\prime}$ be the vertex diagonally opposite to $u_{1}^{\prime}$ in $\operatorname{Cspan}\left(\left\{u_{3}, u_{1}\right\}\right)$. The triple $\left\lfloor u_{1}^{\prime}, u_{2}, u_{3}^{\prime}\right\rfloor$ is equivalent to $\left\lfloor u_{1}, u_{2}, u_{3}\right\rfloor$, it satisfies, $u_{1}^{\prime}, u_{2} \in F_{k}$ (hence we must have $\left.u_{3}^{\prime} \notin F_{k}\right)$, and $\operatorname{Cspan}\left(\left\{u_{1}^{\prime}, u_{2}\right\}\right)$ properly contains $\operatorname{Cspan}\left(\left\{u_{1}, u_{2}\right\}\right)$, contradicting the maximality of $\operatorname{dim} \operatorname{Cspan}\left(\left\{u_{1}, u_{2}\right\}\right)$.

By the minimality of $\mathscr{E}$, for any $i \in\{1,2, \ldots, d-1\}$ the vertices $u_{1}$ and $u_{2}$ cannot be both contained in $A_{i}^{0}$, otherwise the triple $\left\lfloor u_{1}, u_{2}, u_{3}\right\rfloor$ is already a counterevidence in $\left.\left.\mathscr{C}\right|_{F_{1}} \cup \cdots \cup \mathscr{E}\right|_{F_{k-1}}$. Similarly, the last coordinate of $u_{1}$ and $u_{2}$ cannot agree. These considerations show that the vertices $u, v \in F_{k}$ defined by $\phi(u) \stackrel{\text { def }}{=}(1,1, \ldots, 1,0)$ and $\phi(v) \stackrel{\text { def }}{=}(1,1, \ldots, 1,1)$ both belong to $C \operatorname{span}\left(\left\{u_{1}, u_{2}\right\}\right)$. We claim that $\operatorname{Cspan}\left(\left\{u_{3}, u\right\}\right)$ and $\operatorname{Cspan}\left(\left\{u_{3}, v\right\}\right)$ are edges. In fact, as noted above, half of Cspan $\left(\left\{u_{3}, u\right\}\right)$ is contained in $\operatorname{Cspan}\left(\left\{u_{1}, u_{2}\right\}\right)$. It is sufficient to show therefore that $\operatorname{Cspan}\left(\left\{u_{3}, u\right\}\right) \cap \operatorname{Cspan}\left(\left\{u_{1}, u_{2}\right\}\right)$ is zero-dimensional. If not, then it contains a vertex $u^{\prime}$ for which $\phi\left(u^{\prime}\right)$ differs from $\phi(u)$ from exactly one coordinate, say the $j$ th one. When $j=d$ then we get a contradiction by Lemma 8 , when $j \leq d-1$ we get a contradiction by Lemma 9 . Hence $\left\{u_{3}, u\right\}$ is an edge and similarly $\left\{u_{3}, v\right\}$ is an edge. However, then $u_{3}, u$, and $v$ form a triangle in the edge-graph of $\mathscr{E}$, which therefore cannot be bipartite, contradicting Lemma 4 .

Propositions $1-3$ imply the following theorem.

Theorem 6. Every shellable cubical complex of dimension two is well behaved. 
Proof. Take a minimal counterexample $\mathscr{C}$ with shelling $F_{1}, \ldots, F_{k}$. By Lemma 3, the possible types of attachments of $\left.\mathscr{C}\right|_{F_{k}}$ to $\left.\left.\mathscr{C}\right|_{F_{1}} \cup \cdots \cup \mathscr{C}\right|_{F_{k-1}}$ are the following: $(1,0),(2,0),(1,1)$, and $(0,2)$. Types $(1,0)$ and $(2,0)$ are excluded by Proposition 1 , type $(0,2)$ is forbidden by Proposition 2 , and finally type $(1,1)$ is disallowed by Proposition 3.

Finally, we prove the main theorem of this section.

Theorem 7. Let $\mathscr{E}$ be a $(d-1)$-dimensional shellable subcomplex of the boundary complex of a d-dimensional convex cubical polytope $P$. Then $\mathscr{C}$ is well behaved.

Proof. Let $\mathscr{E}$ be a minimal counterexample, and let $F_{1}, F_{2}, \ldots, F_{k}$ be a shelling of $\mathscr{C}$. By the previous results, we may assume $d \geq 4$, and that the type of attachment of $\left.\mathscr{C}\right|_{F_{k}}$ to $\left.\left.\mathscr{C}\right|_{F_{1}} \cup \cdots \cup \mathscr{C}\right|_{F_{k-1}}$ is $(r, 1)$ with $0<r \leq d-3$. Let $H_{1}$ and $H_{2}$ be the only pair of opposite facets of $\partial\left(\left.\mathscr{E}\right|_{F_{k}}\right)$ such that they both belong to $\left.\left.\mathscr{E}\right|_{F_{1}} \cup \cdots \cup \mathscr{E}\right|_{F_{k-1}}$.

Let us take a counterevidence $\left\lfloor u_{1}, u_{2}, u_{3}\right\rfloor$ such that $u_{1}, u_{2} \in F_{k}$ hold, and the dimension of $\operatorname{Cspan}\left(\left\{u_{1}, u_{2}\right\}\right)$ be maximal under these conditions. Let us denote $\operatorname{Cspan}\left(\left\{u_{1}, u_{2}\right\}\right)$ by $\tau_{3}$. By Lemma 7 , the faces $\tau_{i} \stackrel{\text { def }}{=} \operatorname{Cspan}\left(\left\{u_{3}\right\} \cup\left(\tau_{3} \cap H_{i}\right)\right)$ exist for $i=1,2$. As in the proof of Theorem 6 , the maximality of $\operatorname{dim} \operatorname{Cspan}\left(\left\{u_{1}, u_{2}\right\}\right)$ implies that exactly half of the vertices of $\tau_{1}$ or $\tau_{2}$ belong to $\tau_{3}$. Thus we have

$$
\frac{\left|\tau_{1}\right|}{2}=\left|\tau_{1} \cap \tau_{3}\right|=\left|\tau_{3} \cap H_{1}\right|=\frac{\left|\tau_{3}\right|}{2}=\left|\tau_{3} \cap H_{2}\right|=\left|\tau_{2} \cap \tau_{3}\right|=\frac{\left|\tau_{1}\right|}{2}
$$

and so $\tau_{1}, \tau_{2}$, and $\tau_{3}$ have the same dimension (Fig. 2). Let us denote this dimension by $\delta$.

Let $S$ be the affine hull of $u_{3}$ and $\tau_{3}$. It is a $(\delta+1)$-dimensional plane, and it intersects the polytope $P$ in a $(\delta+1)$-dimensional polytope $P^{\prime}$. Clearly, $S$ contains both $\tau_{1}$ and $\tau_{2}$, because half of these faces is a $(\delta-1)$-face of $\tau_{3}$ (and so belongs to $S$ ) and the affine span of $u_{3}$ and $\tau_{i} \cap \tau_{3}$ contains $\tau_{i}(i=1,2)$.

Consider the "pyramid" $Q \stackrel{\text { def }}{=} \operatorname{conv}\left(u_{3}, \tau_{3}\right)$. We may assume that $\operatorname{relint}(Q) \subset \operatorname{int}(P)$ otherwise $Q$ is contained in a face of $\partial(P)$, and so there is a face of $\partial(P)$ containing $\tau_{1}, \tau_{2}$, and $\tau_{3}$. It is easy to convince ourselves, however, that no cube can contain three equidimensional faces with the intersection properties of $\tau_{1}, \tau_{2}$, and $\tau_{3}$ : if half of the vertices of $\tau_{1}$ and $\tau_{2}$ intersected $\tau_{3}$ in opposite halves of $\tau_{3}$, then $\tau_{1} \cap \tau_{2}$ would be empty, and we need $u_{3} \in \tau_{1} \cap \tau_{2}$. The affine hull of $Q$ is $S$.

Without loss of generality we may assume that $u_{1}$ is diagonally opposite to $u_{3}$ in $\tau_{1}$. (If not, we may replace $\left[u_{1}, u_{2}\right\rfloor$ by another diagonal of $\tau_{3}$.) Let $u_{3}^{\prime}$ be the vertex of $\tau_{1} \backslash \tau_{3}$ which is connected to $u_{1}$ by an edge. (In other words, let $u_{3}^{\prime}$ be the vertex diagonally opposite to $u_{3}$ in the face $\tau_{1} \backslash \tau_{3}$.) Let $u_{1}^{\prime}$ be the vertex diagonally opposite to $u_{1}$ in $\tau_{3} \cap \tau_{1}$. Then $u_{1}^{\prime}$ is diagonally opposite to $u_{3}^{\prime}$ in $\tau_{1}$ and so $\left\lfloor u_{1}^{\prime}, u_{2}, u_{3}^{\prime}\right\rfloor$ is equivalent to $\left\lfloor u_{1}, u_{2}, u_{3}\right\rfloor$, hence there is a face containing $u_{3}^{\prime}$ and $u_{2}$. In particular, the line segment connecting $u_{3}^{\prime}$ and $u_{2}$ belongs to $\partial(P)$ and thus it cannot have any common point with $\operatorname{relint}(Q)$. 


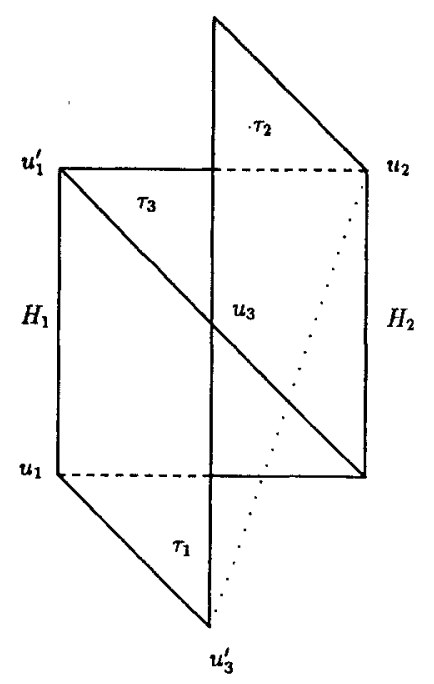

Fig. 2. Illustration to the proof of Theorem 7 .

Consider now those supporting hyperplanes of $Q$ in $S$ which intersect $Q$ in a facet of $Q$. These hyperplanes are $\delta$-dimensional, and with the exception of the affine hull of $\tau_{3}$, they all arise as the affine hull of $u_{3}$ and of a $(\delta-1)$-face of $\tau_{3}$. For each such hyperplane $K$, let us call that half-space of $K$ in $S$ which contains $Q$, the positive half of $K$. If $K$ contains $u_{1}^{\prime}, u_{2}$, and $u_{3}$, then $u_{3}^{\prime}$ is in the strict positive half of $K$. In fact, $K$ then intersects $\tau_{3}$ in a ( $\left.\delta-1\right)$-face of $\tau_{3}$ and so it intersects $\tau_{1} \cap \tau_{3}$ in a $(\delta-2)$-face. $K \cap \tau_{1}$ contains this $(\delta-2)$-face and $u_{3}$, and so $K \cap \tau_{1}$ contains a $(\delta-1)$-face of $\tau_{1}$. Thus $K \cap \tau_{1}$ is equal to this $(\delta-1)$-face, because otherwise $K$ would contain the whole affine hull of $\tau_{1}$ which does not contain $u_{2}$. The $(\delta-1)$-face $K \cap \tau_{1}$ of $\tau_{1}$ contains $u_{1}^{\prime}$ and so it cannot contain $u_{3}^{\prime}$ which is diagonally opposite to $u_{1}^{\prime} \in K$ in $\tau_{1}$.

The only hyperplanes of facets of $Q$ through $u_{2}$ which do not contain both $u_{3}$ and $u_{1}^{\prime}$ are $\operatorname{aff}\left(\tau_{3}\right)$ and aff $\left(\tau_{2}\right)$. The vertex $u_{3}^{\prime}$ cannot be in the strict positive half of both of them, because otherwise the line segment connecting $u_{3}^{\prime}$ with $u_{2}$ would contain a point of relint $(Q)$ close to $u_{2}$. Therefore either $u_{3}^{\prime} \in \operatorname{aff}\left(\tau_{3}\right)$ or $u_{3}^{\prime} \in \operatorname{aff}\left(\tau_{2}\right)$ must hold. Equivalently, we have either $u_{3}^{\prime} \in \tau_{3}$ or $u_{3}^{\prime} \in \tau_{2}$.

Recall that $u_{3}^{\prime} \in \tau_{1} \backslash \tau_{3}$, so $u_{3}^{\prime} \notin \tau_{3}$. Therefore $u_{3}^{\prime}$ must belong to $\tau_{2}$ and so $\tau_{1} \cap \tau_{2}$ contains the $(\delta-1)$-face $\operatorname{Cspan}\left(\left\{u_{3}, u_{3}^{\prime}\right\}\right)$, hence $\tau_{1} \cap \tau_{2}$ must also be $(\delta-1)$-dimensional. We claim that in this case $u_{2}$ is connected to $u_{3}$ by an edge. In fact by what was said above, the line segment connecting $u_{3}^{\prime}$ and $u_{2}$ intersects relint $\left(\tau_{2}\right)$ and so $u_{3}^{\prime}$ and $u_{2}$ are diagonally opposite in $\tau_{2}$, and $u_{3}$ is connected to $u_{2}$ by an edge. Therefore $u_{1}^{\prime}, u_{2}$, and $u_{3}$ form a triangle, contradicting Lemma 4 .

Corollary 6. The boundary complex of a convex cubical polytope is well behaved.

Proof. As a special case of the results shown in [4], the boundary complex of a convex cubical polytope is shellable. Hence we may apply Theorem 7 . 


\section{Edge-Orientable Cubical Complexes}

Definition 16. We call two edges $\{u, v\}$ and $\left\{u^{\prime}, v^{\prime}\right\}$ of a cubical complex $\mathscr{C}$ parallel if there is a facet $F \in \mathscr{C}$ containing $\left\{u, u^{\prime}, v, v^{\prime}\right\}$, and a subfacet $H \subset F$ such that $|\{u, v\} \cap H|=\left|\left\{u^{\prime}, v^{\prime}\right\} \cap H\right|=1$.

We can turn the edge-graph of $\mathscr{C}$ into a directed graph by defining a function

$$
\pi: V \times V \rightarrow\{-1,0,1\}
$$

satisfying the following properties:

(i) $\pi(u, v)=-\pi(v, u)$ holds for all $u \neq v$.

(ii) $\pi(u, v)=0$ if and only if $\{u, v\}$ is not an edge of $\mathscr{C}$.

(When $\pi(u, v)=1$ we say that "the edge points from $u$ toward $v$. ")

We call $\pi$ an orientation of the edge-graph of $\mathscr{C}$ or edge-orientation on $\mathscr{C}$ if it satisfies the following condition: given two parallel edges $\{u, v\}$ and $\left\{u^{\prime}, v^{\prime}\right\}$, a facet $F$ containing these edges and a subfacet $H \subset F$ such that $\{u, v\} \cap H=u,\left\{u^{\prime}, v^{\prime}\right\} \cap H$ $=u^{\prime}$, we have

$$
\pi(u, v)=\pi\left(u^{\prime}, v^{\prime}\right) .
$$

We call $\mathscr{E}$ edge-orientable, if its edge-graph has an orientation $\pi$.

In plain English, edge-orientability means that we can direct the edges of $\mathscr{E}$ such that "parallel edges point in the same direction." As a consequence of Jordan's theorem, the boundary complex of a three-dimensional cubical polytope is edgeorientable. Another often-studied class of cubical polytopes with edge-orientable boundary is the class of cubical zonotopes. For general cubical polytopes in higher dimensions, edge-orientability means that every $(d-2)$-dimensional manifold connecting the midpoints of parallel edges is orientably embedded into the boundary of the polytope. It seems to be intuitively clear to the author that there are convex four-dimensional cubical polytopes, of which the boundary complex contains a "Möbius strip" of three-dimensional cubes, but we leave the verification of this conjecture to the reader.

Conjecture 2. Convex cubical polytopes, of which the boundary complex is not edge-orientable, exist.

The following lemma shows the existence of a labeling for a shellable and edge-orientable complex $\mathscr{E}$ which has important applications.

Lemma 10. Let $\mathscr{C}$ be a shellable and edge-orientable complex of dimension at least two and let $\pi$ be an orientation of the edge-graph of $\mathscr{B}$. Then there is a labeling

$$
\theta: V \rightarrow \mathbb{Z}
$$

such that for every edge $\{u, v\}$ we have

$$
\theta(v)-\theta(u)=\pi(u, v) .
$$


Proof. The proof is analogous to the proof of Lemma 4. Assume $\mathscr{E}$ is a counterexample with a minimal number of facets. Let $F_{1}, F_{2}, \ldots, F_{k}$ be a shelling of $\mathscr{E}$. The complex $\left.\left.\mathscr{C}\right|_{F_{1}} \cup \cdots \cup \mathscr{E}\right|_{F_{k-1}}$ is shellable, and the restriction of $\pi$ provides an edge-orientation on it. Hence, by the minimality of $\mathscr{E}$, there is a labeling $\theta^{\prime}$ on it which satisfies (8) for every pair of vertices of $\left.\left.\mathscr{E}\right|_{F_{1}} \cup \cdots \cup \mathscr{E}\right|_{F_{k-1}}$. On the other hand, it is easy to see that there is a labeling $\theta^{\prime \prime}$ on the cube $\left.\mathscr{C}\right|_{F_{k}}$ : we can take a standard geometric realization $\phi:\left.\mathscr{E}\right|_{F_{k}} \rightarrow[0,1]^{\operatorname{dim}(\mathscr{E})}$, such that the only vertex with no incoming edges in $\left.\mathscr{C}\right|_{F_{k}}$ goes into $(0,0, \ldots, 0)$, and the only vertex with no outgoing edges in $\left.\mathscr{C}\right|_{F_{k}}$ goes into $(1,1, \ldots, 1)$. Then we can set $\theta^{\prime \prime}(v)$ to be the sum of the coordinates of $\phi(v)$ for every $v \in F_{k}$. It is easy to check that this labeling will also satisfy (8) for every pair of vertices of $F_{k}$.

Clearly, if a labeling $\theta$ satisfies (8) in a complex, then the same holds for $\theta+c$, where $c$ is an arbitrary constant. Thus we may assume that we have a $v_{0} \in F_{k} \cap\left(F_{1}\right.$ $\left.\cup \cdots \cup F_{k-1}\right)$ such that $\theta^{\prime}\left(v_{0}\right)=\theta^{\prime \prime}\left(v_{0}\right)$ holds. However, then, as we have observed in the proof of Lemma 4, the edge-graph of the complex $\left.\mathscr{C}\right|_{F_{k}} \cap\left(\left.\left.\mathscr{C}\right|_{F_{1}} \cup \cdots \cup \mathscr{C}\right|_{F_{k-1}}\right)$ is connected. It is easy to see that if $\theta^{\prime}$ and $\theta^{\prime \prime}$ are labelings satisfying (8) in a directed graph $G$, which has a connected graph as the underlying undirected graph, then their difference is constant. Thus, by $\theta^{\prime}\left(v_{0}\right)=\theta^{\prime \prime}\left(v_{0}\right)$, the restriction of $\theta^{\prime}$ to $\left.\mathscr{C}\right|_{F_{k}} \cap\left(\left.\left.\mathscr{E}\right|_{F_{1}} \cup \cdots \cup \mathscr{C}\right|_{F_{k-1}}\right)$ is equal to the restriction of $\theta^{\prime \prime}$ to $\left.\mathscr{E}\right|_{F_{k}} \cap$ $\left(\left.\left.\mathscr{E}\right|_{F_{1}} \cup \cdots \cup \mathscr{E}\right|_{F_{k}-1}\right)$. Therefore we can define

$$
\theta(v) \stackrel{\operatorname{def}}{=} \begin{cases}\theta^{\prime}(v) & \text { when } \quad v \in F_{1} \cup \cdots \cup F_{k-1}, \\ \theta^{\prime \prime}(v) & \text { when } v \in F_{k},\end{cases}
$$

and obtain a labeling for $\mathscr{C}$ that satisfies (8), contradicting our assumption.

Lemma 11. Let $\mathscr{C}$ be a shellable, edge-orientable cubical complex, and let $\pi$ be an edge-orientation of $\mathscr{C}$. Then the transitive closure $<_{\pi}$ of the relation

$$
u<{ }_{\pi}^{\text {def }} v \quad \text { whenever } \quad \pi(u, v)=1
$$

is a partial order on the vertex set $V$ of $\mathscr{B}$.

Proof. We only need to show that there is no sequence of vertices $v_{1}, v_{2}, \ldots, v_{k}$ such that

$$
\pi\left(v_{1}, v_{2}\right)=\pi\left(v_{2}, v_{3}\right)=\cdots=\pi\left(v_{k-1}, v_{k}\right)=\pi\left(v_{k}, v_{1}\right)=1
$$

would hold. If we had such a sequence, then for a labeling $\theta$ satisfying (8) we would have $\theta\left(v_{i+1}\right)=\theta\left(v_{i}\right)+1$ for $i=1,2, \ldots, k-1$, and $\theta\left(v_{k}\right)+1=\theta\left(v_{1}\right)$. However, this would imply

$$
\theta\left(v_{1}\right)=\theta\left(v_{1}\right)+k
$$

a contradiction. 
Definition 17. Let $\mathscr{E}$ be a shellable, edge-orientable cubical complex and let $\pi$ be an edge-orientation of $\mathscr{C}$. We call the partial order described in Lemma 11 the partial order induced by $\pi$, and we denote it by $<_{\pi}$.

We define the triangulation $\Delta_{\pi}(\mathscr{C})$ of $\mathscr{C}$ induced by $\pi$ as follows:

1. We set $V\left(\Delta_{n}(\mathscr{C})\right) \stackrel{\text { def }}{=} \mathrm{V}(\mathscr{C})$.

2. A set $\left\{v_{1}, \ldots, v_{k}\right\} \subseteq V(\mathscr{C})$ is a face of $\Delta_{n}(\mathscr{E})$ if and only if $\operatorname{Cspan}\left(\left\{v_{1}, v_{2}, \ldots, v_{k}\right\}\right)$ exists and $\left\{v_{1}, v_{2}, \ldots, v_{k}\right\}$ is a chain in the partially ordered set $\left(V,<_{\pi}\right)$.

Lemma 12. Given a shellable and edge-orientable cubical complex $\mathscr{C}$ and an edgeorientation $\pi$ of $\mathscr{C}$, we have $\Delta_{\pi}(\mathscr{C})=\Delta_{<}(\mathscr{C})$ for any linear extension $<$ of the partial order $<_{\pi}$.

Proof. Take an arbitrary subset $\left\{v_{1}, \ldots, v_{k}\right\}$ of the vertex set $V$. Witout loss of generality we may assume $v_{1}>\cdots>v_{k}$.

If Cspan $\left(\left\{v_{1}, \ldots, v_{k}\right\}\right)$ does not exist, then $\left\{v_{1}, \ldots, v_{k}\right\}$ does not belong to any of $\Delta_{\pi}(\mathscr{C}), \Delta_{<}(\mathscr{C})$. Thus we may assume that $\operatorname{Cspan}\left(\left\{v_{1}, \ldots, v_{k}\right\}\right)$ exist, and without loss of generality we may even assume that there is no vertex outside $\operatorname{Cspan}\left(\left\{v_{1}, \ldots, v_{k}\right\}\right)$, i.e., $\mathscr{C}$ is a standard $n$-cube $\mathscr{C}^{n}=\operatorname{Cspan}\left(\left\{v_{1}, \ldots, v_{k}\right\}\right)$ for some $n$.

In this special case the statement can be easily shown by induction on $k$. In fact, $\left\{v_{1}, \ldots, v_{k}\right\} \in \Delta_{\pi}(\mathscr{C})$ holds iff we have $\left\{v_{1}, \ldots, v_{k-1}\right\} \in \Delta_{n}\left(\operatorname{Cspan}\left(\left\{v_{1}, \ldots, v_{k-1}\right\}\right)\right)$ and $v_{k}$ is the unique minimal element of $\operatorname{Cspan}\left(\left\{v_{1}, \ldots, v_{k}\right\}\right)$ with respect to the partial order $<_{\pi}$. Similarly, we have $\left\{v_{1}, \ldots, v_{k}\right\} \in \Delta_{<}(\mathscr{E})$ iff $\left\{v_{1}, \ldots, v_{k-1}\right\} \in$ $\Delta_{<}\left(\operatorname{Cspan}\left(\left\{v_{1}, \ldots, v_{k-1}\right\}\right)\right)$, and $v_{k}$ is the unique minimal element of $\operatorname{Cspan}\left(\left\{v_{1}, \ldots\right.\right.$, $\left.\left.v_{k}\right\}\right)$ with respect to the linear order $<$. Observe finally that, $<$ being an extension of $<_{\pi}$, the unique minimal element of $\operatorname{Cspan}\left(\left\{v_{1}, \ldots, v_{k}\right\}\right)$ is the same with respect to both orders.

Recall that a $d$-dimensional pure simplicial complex $\Delta$ is completely balanced if the vertex set of $\Delta$ may be colored with $d+1$ colors such that no two vertices of the same color belong to a common face.

Lemma 13. Let $\mathscr{C}$ be a d-dimensional shellable edge-orientable complex and let $\pi$ be an edge-orientation of $\mathscr{C}$. Then $\Delta_{\pi}(\mathscr{C})$ is a completely balanced simplicial complex.

Proof. As shown in Lemma 10, there is a labeling $\theta$ of the vertices of $\mathscr{E}$ satisfying (8). Color the vertex $v$ with the modulo $(d+1)$ equivalence class of $\theta(v)$. We claim that $\Delta_{\pi}(\mathscr{C})$ becomes a completely balanced complex, with this coloring. In fact, let us take a face $\left\{v_{1}, v_{2}, \ldots, v_{k}\right\} \in \Delta_{n}(\mathscr{E})$. By the definition of the triangulation, there is a facet $F \in \mathscr{C}$ containing $\left\{v_{1}, v_{2}, \ldots, v_{k}\right\}$, and without loss of generality we may assume $v_{1}<_{\pi} v_{2}<_{\pi} \cdots<_{\pi} v_{k}$. It is an easy consequence of (8) that then we have

$$
\theta\left(v_{1}\right)<\theta\left(v_{2}\right)<\cdots<\theta\left(v_{k}\right) \text {. }
$$

The values of $\theta$ on $F$ are $d+1$ consecutive integers, hence no two of the above $\theta\left(v_{i}\right)$ 's can be congruent modulo $(d+1)$, and so $\Delta_{\pi}(\mathscr{E})$ is a balanced complex. 
Remark. The coloring described in the proof of Lemma 13 allows us to give an explicit system of linear parameters for $K[\mathscr{E}]$. In fact, $\left\{\sum_{\operatorname{color}(v)=i} x_{v}: i=1,2, \ldots, d\right.$ $+1\}$ is such a system. We leave the proof to the reader.

\section{The Eisenbud-Green-Harris Conjecture}

Using the Stanley ring of the boundary complex $\mathscr{E}$ of an edge-orientable convex cubical polytope we may construct an interesting example to a conjecture of Eisenbud, Green, and Harris. Before stating the conjecture, let us recall the definition of the h-vector of a graded algebra. It is a well-known fact that the Hilbert series of a Noetherian $\mathbb{N}$-graded algebra $A$ may be written in the following form:

$$
\mathscr{H}(A, t)=\frac{\sum_{i=0}^{l} h_{i} \cdot t^{i}}{\prod_{i=1}^{s}\left(1-t^{e_{i}}\right)},
$$

where $d \stackrel{\text { def }}{=} \sum_{i=1}^{s} e_{i}$ is the Krull-dimension of $A$, i.e., the maximum length of an increasing chain of prime ideals. (See, e.g., [18].)

Definition 18. We call the vector $\left(h_{0}, \ldots, h_{l}\right)$ in (9) the $h$-vector of the graded Noetherian algebra $A$.

In particular, for a simplicial complex $\Delta$ or a cubical complex $\mathscr{E}$ we define the $h$-vector of the simplicial or cubical complex to be the $h$-vector of their Stanley rings.

Now we may formulate the Eisenbud-Green-Harris conjecture as follows. (See Conjecture $\left(V_{m}\right)$ of [7].)

Conjecture 3. Let $I$ be an ideal of a polynomial ring $K\left[x_{1}, \ldots, x_{r}\right]$ which contains a regular sequence of length $r$ in degree two. Then the h-vector of the graded algebra $K\left[x_{1}, \ldots, x_{r}\right] / I$ is the $f$-vector of some simplicial complex.

Example. Let $\mathscr{E}$ be the boundary complex of a $(d+1)$-dimensional convex cubical polytope, and assume that $\mathscr{E}$ is edge-orientable with an edge-orientation $\pi$. Assume furthermore that $K$ is an infinite field. Then the Stanley ring $K(\mathscr{C})$ is a $d$-dimensional Cohen-Macaulay ring, and it contains a linear system of parameters $l_{1}, \ldots, l_{d}$. We claim that the polynomial ring $K\left[x_{v}: v \in V\right] /\left(l_{1}, \ldots, l_{d}\right)$ and the natural image of the face ideal $I(\mathscr{E})$ in this ring provide an example for Conjecture 3.

In fact, by Theorem 7 the face ideal $I(\mathscr{C})$ is generated by homogeneous elements of degree two, and so the same holds for the image $\overline{I(\mathscr{E})}$ of $I(\mathscr{E})$ in $K\left[x_{v}\right.$ : $v \in V] /\left(l_{1}, \ldots, l_{d}\right)$. Thus $\overline{I(\mathscr{C})}$ contains a maximal regular system of parameters in degree two. The factor of $K\left[x_{v}: v \in V\right] /\left(l_{1}, \ldots, l_{d}\right)$ by $\overline{I(\mathscr{C})}$ is Artinian, isomorphic to $K[\mathscr{E}] /\left(l_{1}, \ldots, l_{d}\right)$, and its $h$-vector is equal to the $h$-vector of the cubical complex $\mathscr{E}$ by the Cohen-Maucalay property of $K[\mathscr{E}]$. By Corollary 3 this $h$-vector is also the $h$-vector of any triangulation via pulling the vertices $\Delta_{<}(\mathscr{E})$ of $\mathscr{E}$. By Lemma 12 , whenever we take a linear extension $<$ of the partial order $<_{\pi}$, the simplicial 
complex $\Delta_{<}(\mathscr{E})$ is equal to the simplicial complex $\Delta_{n}(\mathscr{E})$. Thus we are left to show that the $h$-vector of $\Delta_{\pi}(\mathscr{E})$ is the $f$-vector of some other simplicial complex. However, by Lemma 13 the simplicial complex $\Delta_{\pi}(\mathscr{C})$ is comletely balanced, and-being a triangulation of a sphere - it is a Cohen-Macaulay simplicial complex by Corollary 4.4 of [21]. Therefore its $h$-vector is the $f$-vector of another simplicial complex by Corollary 4.5 of [19].

\section{Acknowledgments}

This paper contains the results of Chapter 3 of my thesis [11]. The starting point to the research presented here was given by my adviser Richard Stanley. He was also the one who drew my attention to the Eisenbud-Green-Harris conjecture. I am grateful to Joe Harris and especially to David Eisenbud for several fruitful discussions. Steve Kleiman enlarged my knowledge of commutative algebra, and Bernd Sturmfels gave me essential information about Gröbner bases. Brian Taylor, Richard Ehrenborg, and Laura Anderson gave useful advice. Finally, I wish to express my gratitude to both of my referees for many helpful comments and suggestions. One of them gave a simpler proof to Theorem 4, and the other helped me to improve greatly the presentation of Section 5 .

\section{References}

1. R. Adin, Private communication.

2. S. Balczerzyk and T. Józefiak, Commutative Rings; Dimension, Multiplicity and Homological Methods, Wiley, New York, 1989.

3. T. Becker and V. Weispfenning, Gröbner Bases, Springer-Verlag, New York, 1993.

4. M. Bruggeser and P. Mani, Shellable decompositions of cells and spheres, Math. Scand. 29 (1971), 197-205.

5. W. Bruns and U. Vetter, Determinantal Rings, Lecture Notes in Mathematics, Vol. 1327, Springer-Verlag, Berlin, 1988.

6. C. Chan, Plane trees and $h$-vectors of shellable cubical complexes, SIM J. Discrete Math. 4(4) (1991), 568-574.

7. D. Eisenbud, M. Green, and J. Harris, Some Conjectures Extending Castelnuovo Theory, Preprint.

8. D. Eisenbud and B. Sturmfels: Binomial ideals, submitted to Duke Math. J.

9. B. Grünbaum, Convex Polytopes, Interscience, London, 1967.

10. J. Herzog and N. V. Trung: Gröbner bases and multiplicity of determinantal and Pfaffian ideals, Adv. in Math. 96 (1992), 1-37.

11. G. Hetyei: Simplicial and Cubical Complexes: Analogies and Differences, Ph.D. thesis, MIT, 1994.

12. M. Hochster, Rings of invariants of tori, Cohen-Macaulay rings generated by monomials and polytopes. Ann. of Math. 96 (1972), 318-337.

13. M. Hochster and J. A. Eagon: Cohen-Macaulay rings, invariant theory, and the generic perfection of determinantal lock, Amer. J. Math. 93 (1971), 1020-1058.

14. H. Matsumura, Commutative Ring Theory, Cambridge University Press, Cambridge, 1992.

15. N. Metropolis and G-C. Rota, Combinatorial structure of the faces of the n-cube, SLAM J. Appl. Math. 35(4) (1978), 689-694. 
16. J. Riordan, Combinatorial Identities, Wiley, New York, 1968.

17. E. H. Spanier, Algebraic Topology, McGraw-Hill, New York, 1966.

18. R. P. Stanley, Hilbert functions of graded algebras, Adv. in Math. 28 (1978), 57-83.

19. R. P. Stanley, Balanced Cohen-Macaulay complexes, Trans. Amer. Math. Soc. 249(1) (1979), 139-157.

20. R. P. Stanley, Decompositions of rational convex polytopes, Ann. Discrete Math. 6 (1980), 333-342.

21. R. P. Stanley, Combinatorics and Commutative Algebra, Birkhäuser, Basel, 1983.

22. B. Sturmfels, Gröbner bases of toric varieties, Tôhoku Mat. J. 43 (1991), 249-261.

Received May 26, 1994, and in revised form October 6, 1994. 